\begin{tabular}{|c|c|}
\hline Citation & $\begin{array}{l}\text { F. Greco, P. Barendrecht, L. Coox, O. Atak and W. Desmet ( } 2017) \\
\text { Finite element analysis enhanced with subdivision surface boundary } \\
\text { representations } \\
\text { Finite Elements in Analysis and Design, } 137,56-72 \text {. }\end{array}$ \\
\hline Archived version & $\begin{array}{l}\text { Author manuscript: the content is identical to the content of the published } \\
\text { paper, but without the final typesetting by the publisher }\end{array}$ \\
\hline Published version & https://www.sciencedirect.com/science/article/pii/S0168874X1630556X \\
\hline Journal homepage & https://www.journals.elsevier.com/finite-elements-in-analysis-and-design \\
\hline \multirow[t]{2}{*}{ Author contact } & francesco.greco@kuleuven.be \\
\hline & +393343779731 \\
\hline IR & \\
\hline
\end{tabular}

(article begins on next page) 


\title{
Finite element analysis enhanced with subdivision surface boundary representations
}

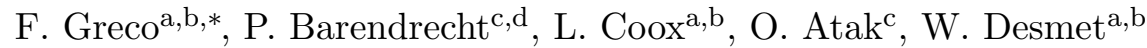 \\ ${ }^{a} K U$ Leuven, Department of Mechanical Engineering, Division PMA, Celestijnenlaan \\ $300 B$ - box 2420, B-3001 Leuven, Belgium. \\ ${ }^{b}$ Member of Flanders Make. \\ ${ }^{c}$ Siemens Industry Software NV, Digital Factory, Product Lifecycle \\ Management - Simulation and Test Solutions, Interleuvenlaan 68, B-3001 Leuven, \\ Belgium. \\ ${ }^{d}$ Johann Bernoulli Institute for Mathematics and Computer Science, University of \\ Groningen, P.O. Box 407, 9700 AK Groningen, The Netherlands.
}

\begin{abstract}
In this work we develop a design-through-analysis methodology by extending the concept of the NURBS-enhanced finite element method (NEFEM) to volumes bounded by Catmull-Clark subdivision surfaces. The representation of the boundary as a single watertight manifold facilitates the generation of an external curved triangular mesh, which is subsequently used to generate the interior volumetric mesh. Following the NEFEM framework, the basis functions are defined in the physical space and the numerical integration is realized with a special mapping which takes into account the exact definition of the boundary. Furthermore, an appropriate quadrature strategy is proposed to deal with the integration of elements adjacent to extraordinary vertices (EVs). Both theoretical and practical aspects of the implementation are discussed and are supported with numerical examples.

Keywords: NURBS enhanced FEM, subdivision surfaces, Catmull-Clark, design-through-analysis
\end{abstract}

\section{Introduction}

From a historical perspective, the development of Computer Aided Design (CAD) and numerical analysis, also referred to as Computer Aided

\footnotetext{
${ }^{*}$ Correspondence to: francesco.greco@kuleuven.be
} 
Engineering (CAE), have followed different paths. The standard used to describe objects in CAD are boundary representations (B-reps). These B-reps are often given by parametric surfaces, commonly based on Non-Uniform Rational B-Splines (NURBS) [1]. On the other hand, in numerical analysis, the reference technique is the Finite Element Method (FEM), where the computational domain is discretized with a mesh of elements. Within each element, polynomial shape functions are constructed for the numerical approximation.

The use of different representations, employed by CAD and FEM, requires the intermediate step of mesh generation which, in many applications, can drastically slow down the entire analysis process. In addition, some geometrical features from the original CAD model may be lost in the FEM representation. As any design-through-analysis process would clearly benefit from a better integration of these two fields, uniting CAD and FEM has become an active topic of research. Major contributions towards this goal are represented by Isogeometric Analysis (IgA) [2, 3] and the NURBSEnhanced Finite Element Method (NEFEM) $[4,5,6,7]$.

The key idea of IgA is to use the same basis functions for both the geometric representation and the numerical analysis. However, limitations arise when the framework is extended to analysis on volumes - whereas a boundary representation is sufficient for CAD purposes, a trivariate parametrization is required for the analysis. This parametrization is not straightforward to construct for complexly shaped objects because of the tensor product nature of NURBS discretizations. In addition, many CAD models are a collection of trimmed NURBS patches that, in general, cannot be stitched together without some small gaps or overlaps occurring at their interfaces.

In order to overcome the limitations imposed by the tensor product structure, other parametric boundary representations have been considered. One of these methods is represented by subdivision surfaces [8, 9], allowing designers to model objects of arbitrary topology as a single watertight surface. Many subdivision schemes produce surfaces that can be numerically evaluated at arbitrary parameter values [10]. Subdivision surfaces were already used for numerical analysis before $\operatorname{IgA}$ was introduced [11, 12]. More recent publications consider analysis on subdivision solids [13] and the local refinement of subdivision splines [14].

Another option is to use T-splines, which can be seen as a generalization of NURBS. The original T-splines [15] were developed to merge two NURBS surfaces defined using different knot vectors (resulting in T-sections in the mesh, hence the name T-splines). Later, they were combined with subdivision surfaces into T-NURCCS [16] using the NURSS framework [17]. More 
recent versions of T-splines have replaced the subdivision approach around irregular points in the control net (referred to as extraordinary vertices in subdivision terminology or star points in T-spline terminology) by $G^{1}$ biquartic patches [18]. An interesting property of T-splines is that they inherently allow local refinement of the control net. IgA based on T-splines [19] has developed considerably over the last few years and achieved several notable results, including the trivariate parametrization of genus-zero objects [20]. The subset of $\mathrm{T}$-splines used in IgA are referred to as analysis-suitable T-splines [21].

In parallel to the development of IgA, NEFEM is another methodology that aims at reducing the gap between CAD and CAE. In the NEFEM approach, the shape functions are defined in the physical space and the exact boundary representation is taken into account in the numerical integration, due to a special volumetric mapping that includes the NURBS parametrization in its formulation [4]. In contrast to IgA, where the trivariate parametrization of complex shapes is one of the main issues, the mesh generation procedure used in the NEFEM framework follows those of standard FEM, paying special attention to capturing small geometric features [5]. The only condition that is assumed in the NEFEM literature is watertightness ( $G^{0}$ continuity) at the interface of the different surface patches [7]. Unfortunately, as mentioned above, complicated CAD models often present some small inconsistencies at such interfaces, which results in complications with regard to FEM mesh generation. Although some strategies, often referred to as cosmetics, are available to improve the original CAD model and facilitate mesh generation, these processes are rarely automatic and require, in most cases, the supervision of the analyst $[22,23]$.

Motivated by these issues, and taking into account the potential showed by subdivision surfaces in IgA [24, 25, 26, 27, 28, 29], we extend the concept of NEFEM to subdivision surfaces. The main advantage of the approach is that the mesh can be automatically generated given a subdivision surface modeled in a CAD environment. Like in the case of NEFEM, the exact geometry is considered in the analysis and the original CAD representation is preserved if p-refinement is applied to the FEM approximation. This is not the case in standard isoparametric finite elements, where the error introduced in the discretization remains also after p-refinement, unless a new mesh is created. At the same time, if h-refinement is required, the generation of a new mesh is facilitated by the watertightness of the surface. In this way, even if a mesh is still used, the conversion between the CAD and the FEM models is no longer a bottleneck.

Although many subdivision algorithms are available, in this work we 
limit our attention to the Catmull-Clark scheme [30], which is the most widely used scheme in both computer graphics and IgA. The outline of the paper is as follows. An overview of subdivision surfaces, with emphasis on the Catmull-Clark scheme, is presented in Section 2. Section 3 describes the procedure employed for the mesh generation, while the NEFEM formulation and its extension to subdivisions surfaces are considered in Section 4. It includes a strategy to handle numerical integration around extraordinary vertices in the surface mesh. Section 5 discusses various numerical examples. Finally, some concluding remarks are given in Section 6.

\section{Subdivision surfaces}

Subdivision surfaces are a powerful tool for designing objects of arbitrary topology [31]. While they represent the leading technology for modeling freeform shapes in animated movies [32] and are a common modeling primitive for $3 \mathrm{D}$ games, the use of subdivision surfaces in CAE is still rather limited. The main reason for this is that most CAD models used for CAE are based on NURBS. This standard allows for the design of models including exact conical sections, something which can only be approximated by traditional subdivision schemes. However, with the advent of NURBS-compatible subdivision surfaces [33] and the possibility to convert trimmed NURBS surfaces to subdivision surfaces [34], the gap between these two standards is closing rapidly. Integrating subdivision surfaces in a CAD/CAE environment has become a topic of active research [35].

Similar developments can be seen in commercial CAD/CAE software packages. Notable examples include the introduction of the Realize Shape environment in NX 9, the Mesh Modeler in AutoCAD 2010, the Imagine \& Shape module in CATIA 5 and the Freestyle extension in PTC CREO, which are all based on subdivision surfaces.

The use of subdivision surfaces in CAE was first proposed in [11]. Other work in a similar framework, by then referred to as IgA, includes $[24,25$, 26, 27, 28]. Solving partial differential equations (PDEs) on subdivision volumes, as opposed to solving PDEs on subdivision surfaces, is a direction of research only just initiated $[13,36]$. It is here that we see the added value of extending the concepts of NEFEM to subdivision surfaces, as it facilitates the numerical analysis on volumes bounded by a subdivision surface.

In this paper we focus on the Catmull-Clark subdivision scheme [30], which is described in the remainder of this section. Combining the approach discussed in this paper with other subdivision schemes based on box-splines [37] is straightforward. 


\subsection{Mesh refinement and smoothing}

From a designer's point of view, a Catmull-Clark subdivision surface can be interpreted as the result of indefinitely refining and smoothing an initial mesh $\mathcal{M}_{0}$. Such a mesh, also referred to as the control net, consists of vertices $\mathcal{V}$, edges $\mathcal{E}$ and quadrilateral faces $\mathcal{F}$.

The refinement and smoothing rules are represented by a set of affine combinations referred to as stencils, indicating how vertices in a mesh $\mathcal{M}_{i}$ should be weighted in order to obtain the mesh $\mathcal{M}_{i+1}$. Repeatedly applying the set of stencils to an initial mesh $\mathcal{M}_{0}$ yields a sequence of meshes $\mathcal{M}_{0}, \mathcal{M}_{1}, \ldots, \mathcal{M}_{i}, \ldots, \mathcal{M}_{\infty}$. Careful selection of the stencils results in the convergence of this sequence to a smooth surface $\mathcal{M}_{\infty}$ which is referred to as the limit surface. Figure 1 illustrates a couple of steps of the CatmullClark scheme. Note that for graphical applications it is often sufficient to subdivide only a few times - the actual limit surface might not be relevant for such purposes.
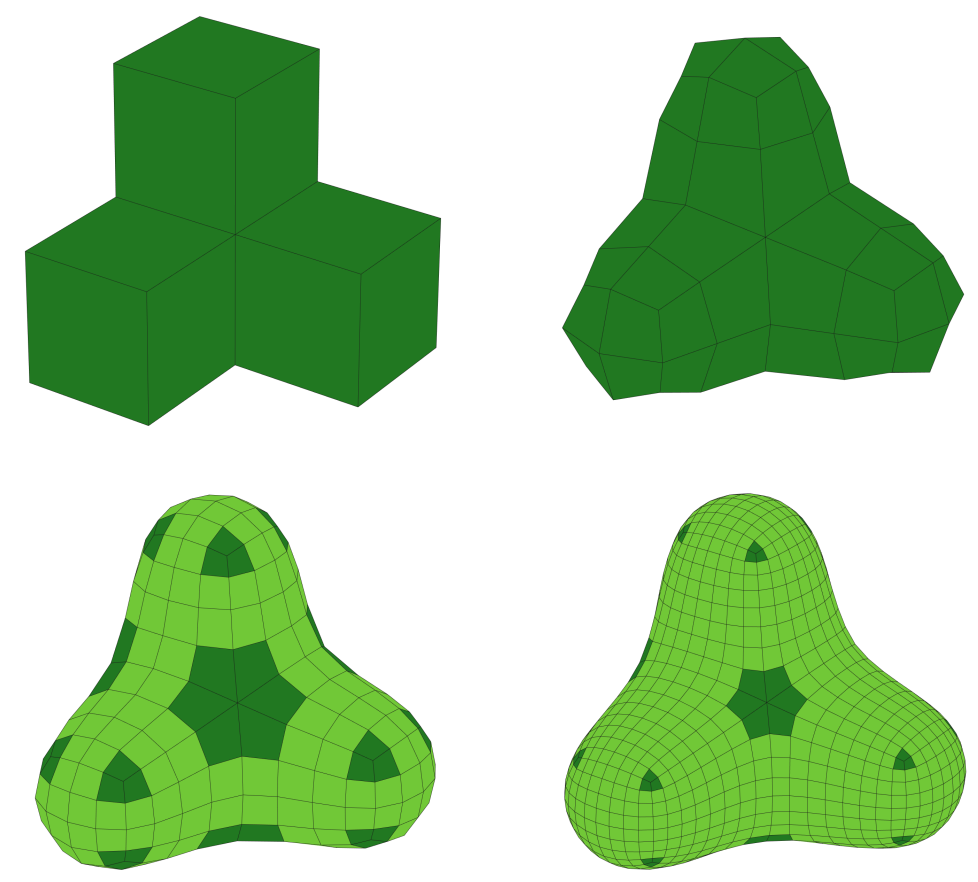

Figure 1: A few steps of the Catmull-Clark subdivision scheme applied to an initial mesh $\mathcal{M}_{0}$ (top left). Faces containing one or more extraordinary vertices are highlighted in dark green. 


\subsection{Masks and stencils}

From a mathematical point of view, the Catmull-Clark scheme is a generalization of midpoint knot-refinement for uniform bicubic B-spline surfaces. In the univariate setting, uniform B-spline basis functions can be defined using a knot-vector $\Xi$. This knot-vector is chosen to be a set of consecutive integers, in other words, $\Xi=[j, j+1, \ldots, k] \subset \mathbb{Z}$. Midpoint knot-refinement consists in inserting the relevant half-integers $\mathbb{Z}+\frac{1}{2}$ into the knot-vector. Repeating this process $i$ times results in a knot-vector $\Xi \subset \mathbb{Z} / 2^{i}$.

Uniform B-splines defined on $\mathbb{Z} / 2^{i}$ can be composed of scaled, shifted versions of themselves, each version scaled by a coefficient [38]. In this context, scaled refers to the smaller versions of the uniform B-splines defined on $\mathbb{Z} / 2^{i+1}$. The concept is illustrated in Figure 2 for the uniform cubic Bspline $N_{4}(t)$, where the subscript indicates the order (defined as degree +1 ) of the B-spline.

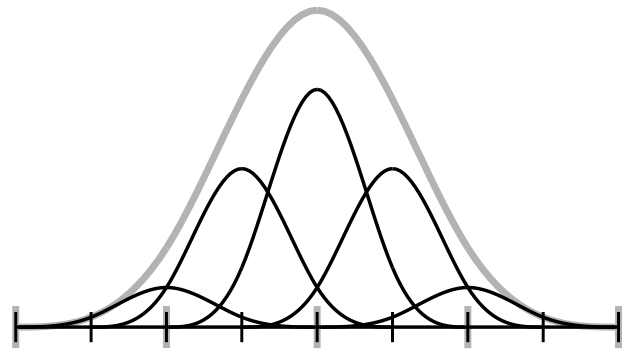

Figure 2: The uniform cubic B-spline $N_{4}(t)$ (grey) expressed as the weighted sum of scaled, shifted versions of itself (black).

When written out, we obtain the expression

$N_{4}(t)=\frac{1}{8} N_{4}(2 t)+\frac{4}{8} N_{4}(2 t-1)+\frac{6}{8} N_{4}(2 t-2)+\frac{4}{8} N_{4}(2 t-3)+\frac{1}{8} N_{4}(2 t-4)$.

The set of coefficients in eq. (1) is called the subdivision mask of the spline. It is common practise to omit the denominator of the coefficients, as they sum to 2 for binary subdivision (in general, the sum is equal to the arity of the subdivision scheme [39]). Division by 8 is therefore implied, and we can write the mask as

$$
\begin{array}{lllll}
1 & 4 & 6 & 4 & 1
\end{array} \text {. }
$$

Stencils associated with a subdivision scheme can be extracted from its mask. For a binary subdivision scheme, the sequence of every other 
coefficient in the mask is a stencil, resulting in the two stencils $\left[\begin{array}{lll}1 & 6 & 1\end{array}\right] / 8$ and $\left[\begin{array}{ll}4 & 4\end{array}\right] / 8$. Note that both stencils indeed represent affine combinations. Any uniform cubic B-spline curve can now be subdivided by applying these stencils to its control polygon; the first stencil updates existing vertices, whereas the second one introduces new vertices. An example is illustrated in Figure 3.
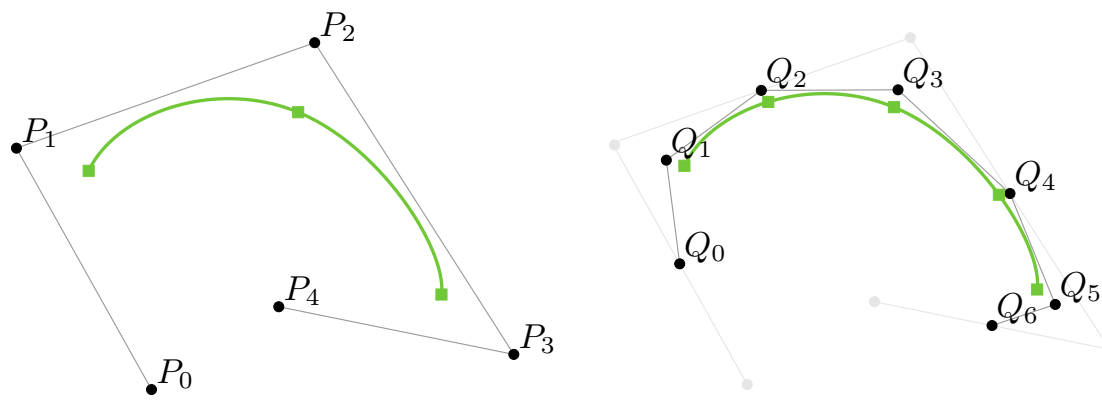

Figure 3: Application of the two stencils associated with the univariate uniform cubic B-spline to a control polygon, resulting in new control points $Q_{0}=\frac{4}{8} P_{0}+\frac{4}{8} P_{1}, \quad Q_{1}=\frac{1}{8} P_{0}+\frac{6}{8} P_{1}+\frac{1}{8} P_{2}, \ldots, Q_{6}=\frac{4}{8} P_{3}+\frac{4}{8} P_{4}$. Note that the segments of the curve directly correspond to edges of the control polygon.

Extending the concept to the tensor-product setting is straightforward. Taking the tensor product of the univariate mask with itself results in the bivariate mask

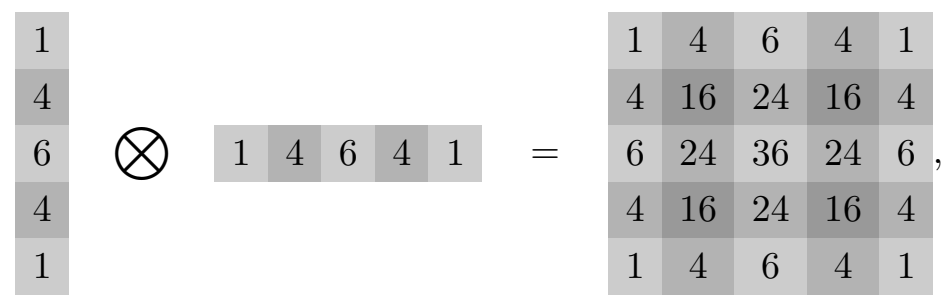

which we express symbolically as

$$
\begin{array}{c|c|c|c|c}
c_{4} & d & b_{4} & d & c_{4} \\
d & f & e & f & d \\
b_{4} & e & a_{4} & e & b_{4} \\
d & f & e & f & d \\
c_{4} & d & b_{4} & d & c_{4}
\end{array}
$$


The regular stencils can be extracted from the mask by selecting every other entry from every other row. They are highlighted in different shades in (4) and are visualized in Figure 4.

At this point we have derived subdivision for uniform bicubic B-spline surfaces. However, as shown in Figure 1, vertices in a quadrilateral mesh can be connected to an arbitrary number $n \geqslant 3$ of edges. The number of edges incident with a vertex $\mathcal{V}$ is referred to as the valence of the vertex. In this setting, vertices with a valence $n \neq 4$ are called extraordinary vertices (EVs).

Designing a stencil to update EVs can be done in different ways. The original paper [30] by Catmull and Clark used an empirical approach, whereas [40] used a more analytical method to derive the coefficients $a_{n}, b_{n}$ and $c_{n}$ for the irregular stencil (see Figure 4, right).
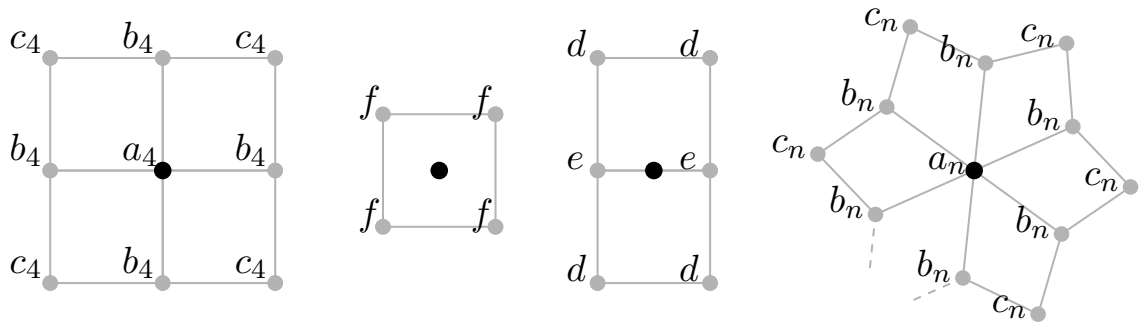

Figure 4: Stencils for the Catmull-Clark scheme. Existing vertices are updated (left and right) whereas new vertices are created for every face (centerleft) and edge (center-right). Both updated and new vertices are indicated in black. The stencil on the right is an irregular stencil.

Although various improvements have been suggested (see e.g. [41] and the references therein), the rules proposed by Catmull and Clark are still the most popular ones. The stencil coefficients can be expressed as simple expressions valid for every valence $n \geqslant 3$ :

$$
a_{n}=1-\frac{7}{4 n}, \quad b_{n}=\frac{3}{2 n^{2}}, \quad c_{n}=\frac{1}{4 n^{2}} .
$$

We now have a complete set of stencils and can apply Catmull-Clark subdivision to any closed quadrilateral mesh. Figure 1 shows several applications of the stencils.

\subsection{Parametric evaluation of the limit surface}

The control net of a B-spline surface has the same topology as a rectangular grid, thereby severely limiting the shapes (e.g. a plane, a cylinder 
without caps, or a torus) that can be modeled with it. The main advantage of subdivision surfaces is that control nets of arbitrary manifold topology can be used, allowing the design of a wide range of freeform surfaces.

Looking at Figure 1 we see that increasingly large parts (indicated in bright green) of the control net match the regular topology of cubic B-spline surfaces as subdivision proceeds. This means that parts of the limit surface associated with these regions can be readily evaluated. Each quadrilateral face $\mathcal{F}_{i}^{k}$, where $i$ refers to the subdivision iteration and $k \in[0,|\mathcal{F}|-1]$ indicates the index of the face, corresponds to a surface patch $\mathcal{S}_{i}^{k}(u, v)$ parameterized on the unit square. Faces containing only regular vertices define a bicubic B-spline patch, see Figure 5 (left).
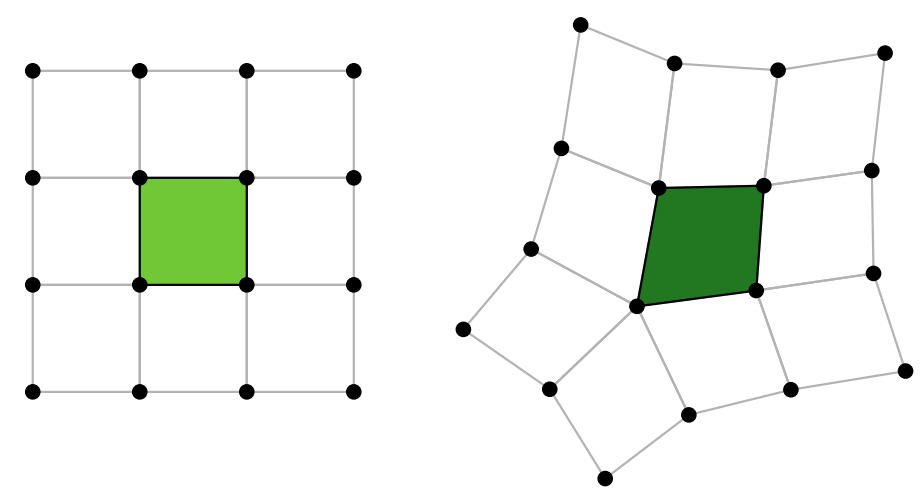

Figure 5: Control nets of a uniform bicubic B-spline patch (left) and an irregular surface patch (right).

The remaining regions (indicated in dark green in Figure 1) of the meshes are all incident with EVs. Note that subdivision does not increase the number of EVs. For $\lim _{i \rightarrow \infty} \mathcal{M}_{i}$ these regions only contain the original EVs from $\mathcal{M}_{0}$ (converged to their limit positions).

The evaluation of a patch $\mathcal{S}_{i}^{k}(u, v)$ associated with a face $\mathcal{F}_{i}^{k}$ containing an EV deserves special attention. In this work, we use the approach proposed by Stam [10]. As illustrated in Figure 6, applying virtual subdivision (i.e. subdivision in memory) around the EV, the patch to be evaluated is split into four sub-patches,

$$
\mathcal{S}_{i}^{k}=\bigcup_{q=0}^{3} \mathcal{S}_{i+1}^{4 k+q}
$$

where $\mathcal{S}_{i+1}^{4 k}$ corresponds to the bottom left patch (indicated in dark green). 
After one step of virtual subdivision, three of these sub-patches can be evaluated as uniform bicubic B-spline patches (Figure 5, left), whereas the remaining sub-patch $\mathcal{S}_{i+1}^{4 k}$ is irregular like the original patch $\mathcal{S}_{i}^{k}$.
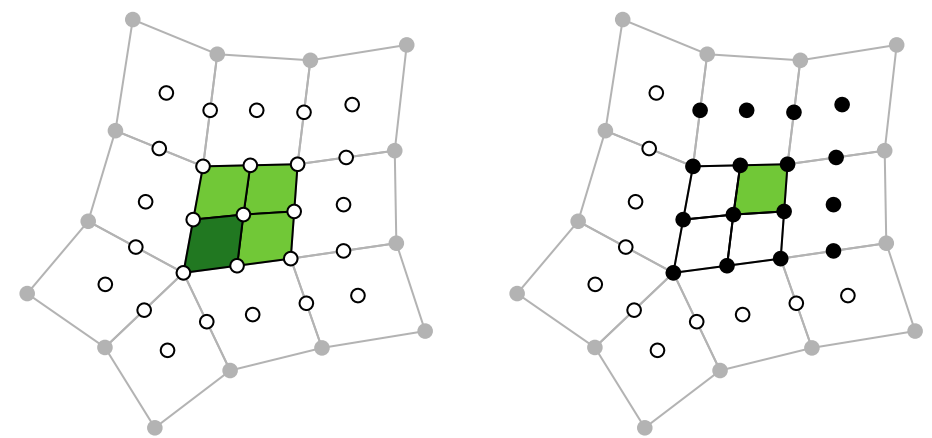

Figure 6: Virtual subdivision around EVs allows three of the four subpatches to be evaluated (left). The control points (indicated in black) that influence one of these regular sub-patches are a subset of the vertices obtained through virtual subdivision (right).

By repeating the process, more layers of ever smaller sub-patches can be evaluated. This is depicted in Figure 7. Note that for each iteration $i \in[1, \infty)$, the set of $3 n$ sub-patches $S_{i}^{k}(u, v)$ around the EV composes a spline ring (see Figure 8).
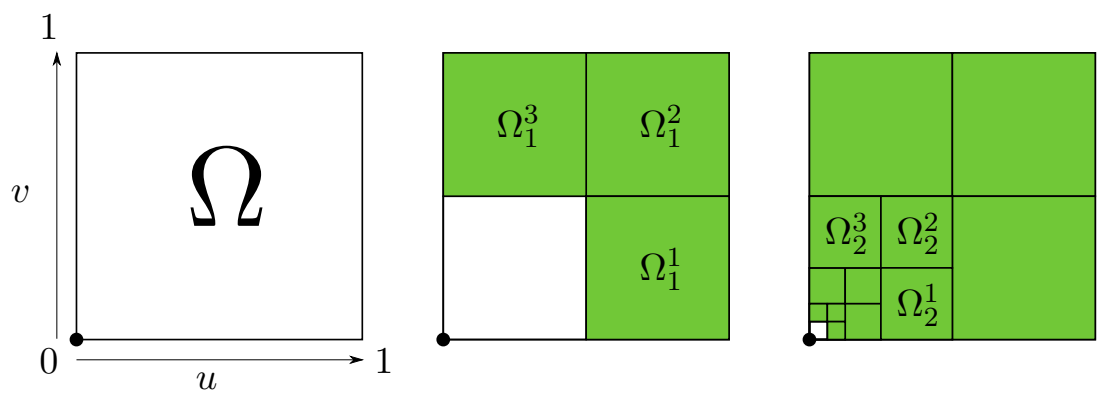

Figure 7: A surface patch associated with a face containing an EV is composed of an infinite number of uniform bicubic B-spline patches (all connecting with $G^{2}$ continuity). It is parameterized on the unit square $\Omega$. The limit position of the $E V(\bullet)$ corresponds to the bottom left corner of $\Omega$.

The limit position of the EV corresponds to $(u, v)=(0,0)$. As it would take an infinite number of virtual subdivision steps to get to this position, it cannot be evaluated using this approach. However, special limit stencils can 
be derived to evaluate the position and unit tangent vectors at this position [42].

Combining the above observations allows us to define a Catmull-Clark subdivision surface as the union of uniform bicubic B-spline patches and the infinite sequences of spline rings around the EVs, including the limit positions of the EVs (see Figure 8).
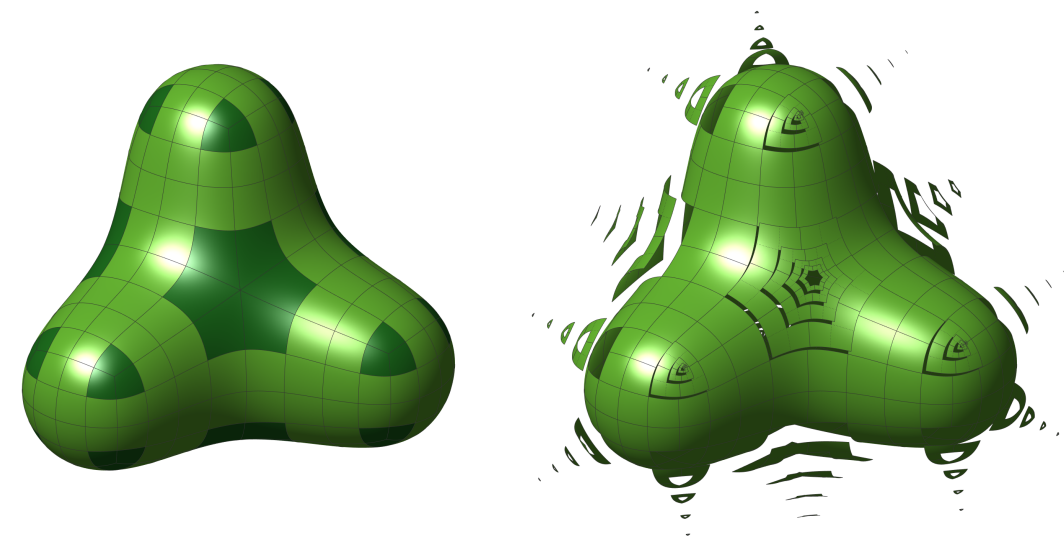

Figure 8: Left: the limit surface illustrated for $\mathcal{M}_{2}$ (see Figure 1). Patches incident with an $E V$ are highlighted in dark green. Right: the same limit surface can be interpreted as the union of bicubic B-spline patches and the infinite sequences of spline rings around the EVs (spline rings offset along the normals of the EVs for visualisation).

There are various techniques [31] that can be used to show that the coefficients in eq. (5) result in curvature-continuous $\left(G^{2}\right)$ limit surfaces, except at the limit positions of the EVs, where the surfaces are merely tangent-plane continuous $\left(G^{1}\right)$.

\section{Mesh generation}

The possibility to work with a single watertight surface given by the subdivision framework is a significant advantage in the automation of the mesh generation process. In this section, the procedure employed to obtain a mesh suitable for the NEFEM implementation is described in detail.

The topic of 3D mesh generation has been widely studied in the FEM literature. We refer to [43] for a recent overview. In the NEFEM approach, tetrahedral elements are considered and, if necessary, the order is increased 
via p-refinement. This simplifies the process compared to the case of using hexahedra.

In this work, we adopt the approach introduced by Persson [44], which considers an analogy between the mesh and a truss structure and computes the position of the nodes by solving a force equilibrium. This method is implemented in the DistMesh generator for MATLAB [45] and is based on an implicit definition of the geometry. By extending it to subdivision surfaces the idea of the force equilibrium is maintained but some modifications are introduced.

The method of the equilibrium can be applied either to a 3D distribution of nodes and its tetrahedral mesh, that is properly shaped into the boundary given by the surface, or to a triangulation defined on the $2 \mathrm{D}$ manifold represented by the surface. The former approach directly produces the final mesh to be used for the analysis, but in three dimensions some sliver elements can be present in the mesh [45]. On the other hand, considering only the triangulation of the surface always provides high quality meshes. Therefore, in this work, the latter approach is preferred, using the force equilibrium only to compute a triangulation on the surface. This triangulation is subsequently passed as an input to a standard mesh generator to obtain the 3D tessellation. In fact, in the NEFEM formulation, nothing changes for the elements in the interior of the domain, though special care is required for those elements that have a curved face or edge on the boundary. In this case, in addition to the physical position of the nodes, the parametric coordinates of these nodes should be determined and stored, as this information is needed in the NEFEM implementation. The mesh generator employed for the numerical examples shown below is the freely available package TETGEN [46].

Before summarizing the procedure to obtain a triangulation of the surface, a remark on the mesh size is necessary. It is a common FEM practice to consider an adaptive approach such that small elements are used in correspondence with small geometrical features. In the implementation using subdivision surfaces, the size of the control net $H(\mathbf{x})$ is directly related to that of these features. It is therefore a reasonable assumption to consider that the size of the FEM mesh $h(\mathbf{x})$ is smaller than $H(\mathbf{x})$. As we will see further on, this also facilitates the implementation.

The meshing process starts with the construction of an initial mesh, whose nodes lay on the limit surface. The easiest way to obtain this mesh is to apply one or more subdivision steps to the initial quadrilateral control net that defines the geometry. Then, using limit stencils, the new control vertices are projected onto the limit surface, preserving the topology given 


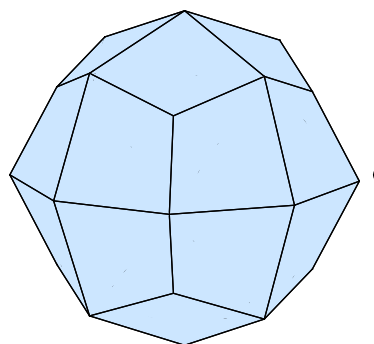

(a)

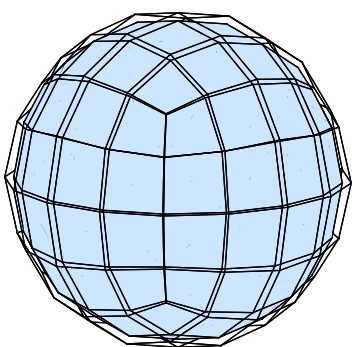

(b)

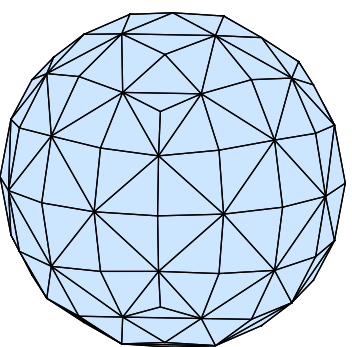

(c)

Figure 9: The generation of the initial mesh for a Catmull-Clark model approximating a sphere. A subdivision step is applied to the initial quadrilateral mesh (a) and the projection of the vertices on the surface is considered (b). The triangular mesh is constructed by splitting the quadrilaterals into two parts (c).

by the control net. Finally, a triangular mesh is obtained by splitting the quadrilaterals into two parts, choosing randomly one of their diagonals.

If the subdivision algorithm is applied more times, a mesh with a higher number of elements is obtained. This procedure, outlined in Figure 9, is particularly efficient for the generation of uniform meshes, or in the case where a constant ratio between $H(\mathbf{x})$ and $h(\mathbf{x})$ is required.

On the other hand, if a given number of nodes or a particular distribution of $h(\mathbf{x})$ have to be imposed, the initial FEM mesh can be generated directly on the quadrilateral control net and then projected on the limit surface. This procedure is outlined in Figure 10. In both cases, we require that the limit positions of the EVs are nodes in the initial mesh.

After the initial mesh has been created, the force equilibrium method described in [45] is applied. In particular, the edges of the mesh are considered as bars of length $l$ and a force is computed according to the following relation:

$$
f(l, h(\mathbf{x}))= \begin{cases}k(h(\mathbf{x})-l) & \text { if } l<h(\mathbf{x}), \\ 0 & \text { if } l \geq h(\mathbf{x}),\end{cases}
$$

where $h(\mathbf{x})$ is the desired length of the edges. This relation considers only a repelling force between the nodes in order to help them spread out over the whole geometry. The value of $h(\mathbf{x})$ can be either specified locally or computed considering a normalized element size function. These values are usually slightly increased in order to facilitate the convergence [45]. Once 


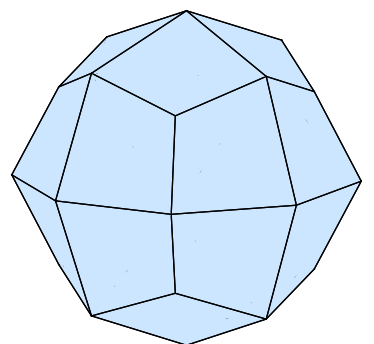

(a)

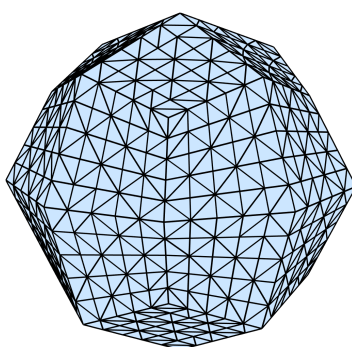

(b)

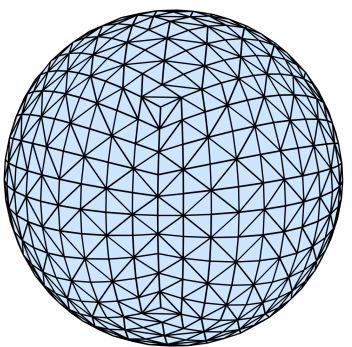

(c)

Figure 10: The generation of the initial mesh for a Catmull-Clark model approximating a sphere. In this case the size of the mesh is imposed. The triangular mesh is created directly on the quadrilateral control net (b) and is subsequently projected onto the limit surface (c).

the repelling forces are computed, the position of the nodes is updated introducing an artificial time dependence:

$$
\boldsymbol{p}_{n+1}=\boldsymbol{p}_{n}+\boldsymbol{F}\left(\boldsymbol{p}_{n}\right) \Delta t
$$

where $\boldsymbol{p}_{i}$ is the position of the nodes at a given (artificial) time step $i$ and $\boldsymbol{F}\left(\boldsymbol{p}_{i}\right)$ is a vector containing, for each node, the sum of all the contributions of the edges incident with the node, computed using eq. (7). We note that, the higher the value of $k$ used to compute $\boldsymbol{F}\left(\boldsymbol{p}_{i}\right)$ is, the lower the value of $\Delta t$ should be for the stability of the method. In particular, the values $k=1$ and $\Delta t=0.2$ suggested in [45] are used in this work.

Due to the effect of the repelling forces, the nodes do not lie on the limit surface anymore after the update and need to be projected back. In order to find the exact projection, a system of nonlinear equations that involves the second derivatives of the surface should be solved [47]. However, this operation would be rather time consuming when repeated for every node and for every iteration. While in [45] implicit surfaces and the relative distance functions are considered, in our implementation with subdivision surfaces the projection of the points is approximated by considering the following linearization of the displacements:

$$
\delta \boldsymbol{p}=\frac{\partial \boldsymbol{p}}{\partial u} \delta u+\frac{\partial \boldsymbol{p}}{\partial v} \delta v .
$$


Thus, assuming $\delta \boldsymbol{p}=\boldsymbol{F}\left(\boldsymbol{p}_{n}\right) \Delta t$, the following system is obtained:

$$
\left\{\begin{array}{l}
\frac{\partial \boldsymbol{p}_{x}}{\partial u} \delta u+\frac{\partial \boldsymbol{p}_{x}}{\partial v} \delta v=\delta \boldsymbol{p}_{x} \\
\frac{\partial \boldsymbol{p}_{y}}{\partial u} \delta u+\frac{\partial \boldsymbol{p}_{y}}{\partial v} \delta v=\delta \boldsymbol{p}_{y} \\
\frac{\partial \boldsymbol{p}_{z}}{\partial u} \delta u+\frac{\partial \boldsymbol{p}_{z}}{\partial v} \delta v=\delta \boldsymbol{p}_{z}
\end{array}\right.
$$

where the unknowns are the variations of the parametric coordinates of the nodes. After solving system (10) in a least square sense, the parametric coordinates are updated. This operation only involves the first derivatives of the surface and the solution of an overdetermined system of linear equations. At this point, the physical position of the nodes $\boldsymbol{p}_{n+1}$ is computed from the new parametric coordinates, with the exception of the nodes in correspondence of EVs that remain constrained to their position. Since the number of EVs is limited over the surface, this constraint does not influence the quality or the characteristics of the final mesh but, as we will see in Section 4, facilitates the implementation of the extended NEFEM.

Before initiating the next iteration, the connectivity between the nodes has to be updated. In this work, the procedure described in [44], that is implemented in the DistMesh toolbox, is employed. The process is iterated until an equilibrium configuration is reached, where the nodes are not subject to significant displacements anymore.

The convergence process for the initial mesh in Figure 10 is illustrated in Figure 11. In Figure 12 two meshes with different levels of refinement for the subdivision model in Figure 1 are shown, while some meshes with a non uniform value of $h(\mathbf{x})$ are shown in Figure 13.

\section{Subdivision Enhanced FEM}

As described in Section 2, every Catmull-Clark surface can be interpreted as the union of cubic B-spline patches and the infinite sequences of spline rings around the EVs. On extending the NEFEM concept to subdivision surfaces, nothing changes with regard to the regular patches. However, a special treatment is required around the EVs.

\subsection{Curved elements on the surface}

Before looking at the details, it is worth remarking that the NEFEM treatment is restricted to the elements that have at least an edge or a face on 


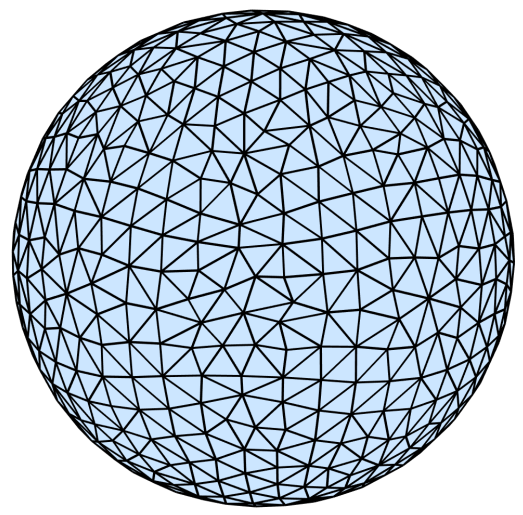

(a)

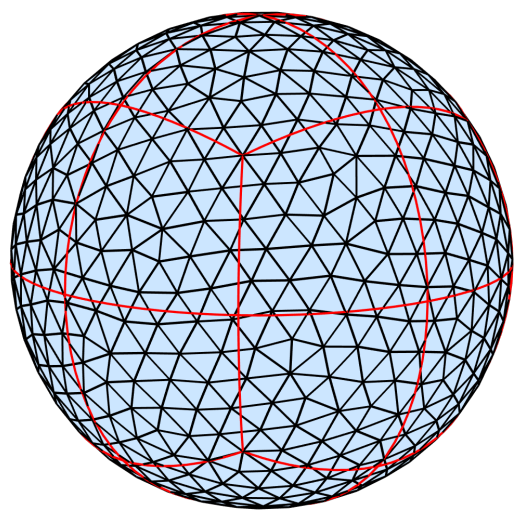

(c)

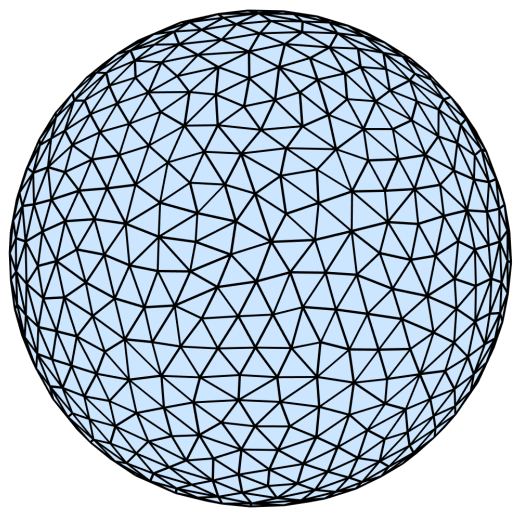

(b)

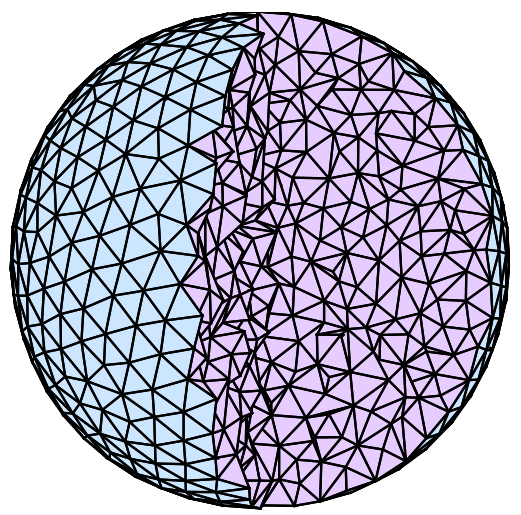

(d)

Figure 11: Two intermediate iterarions of the force equilibrium method applied to the initial mesh in Figure $10(a-b)$; the final mesh with the red lines defining the different Catmull-Clark patches (c) and the $3 D$ mesh generated with TetGen (d). 


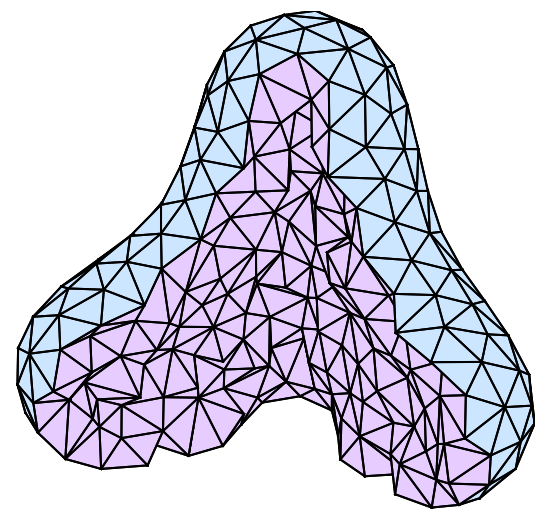

(a)

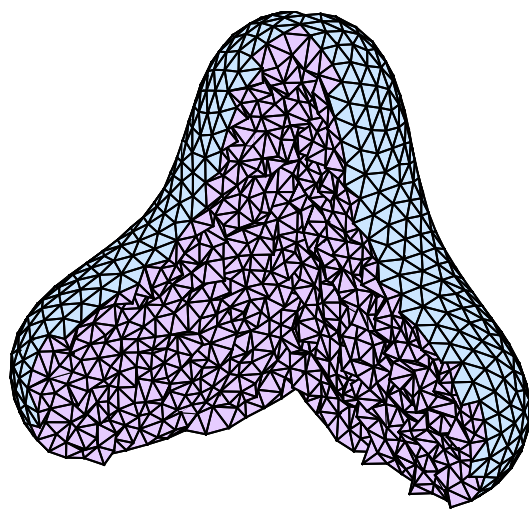

(b)

Figure 12: Two meshes with different levels of refinement for the subdivision model in Figure 1.

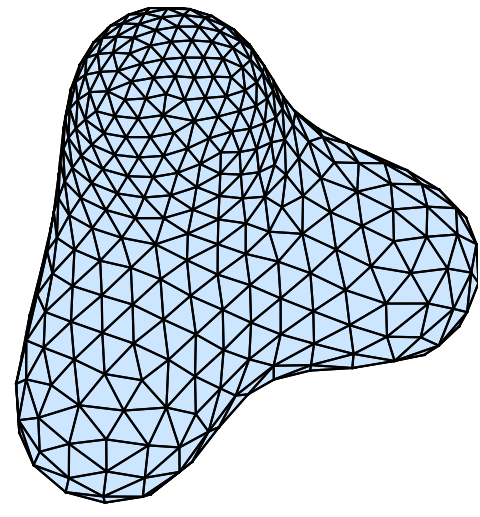

(a)

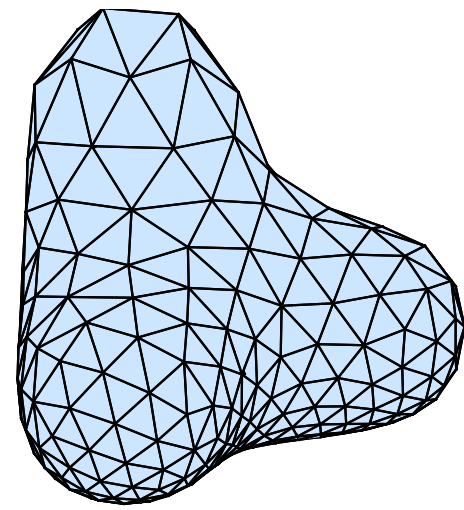

(b)

Figure 13: Two meshes with a non-uniform size $h(\mathbf{x})$ for the subdivision model in Figure 1. 


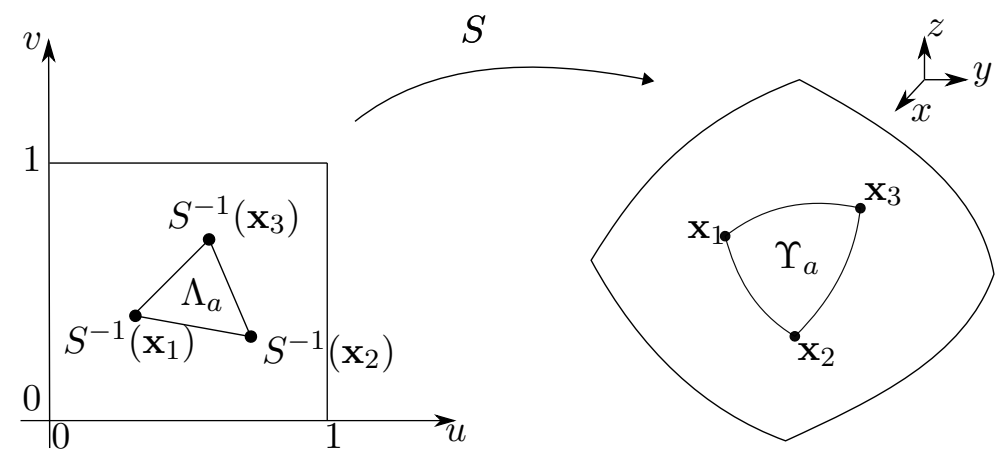

Figure 14: Mapping from a straight-sided triangle in the parametric space to a curved triangle in the physical space.

the boundary. A standard FEM implementation is employed for the interior elements (including those with only a node on the surface). In particular, applying the method described in Section 3 for the mesh generation, three types of elements are possible on the boundary:

- elements with one curved face

- elements with one curved edge

- elements with more than one curved edge or face.

According to [7], elements in the latter category can be split into subelements, effectively reducing the list of categories to the first two types.

At first, we will assume that any element belongs to a single bicubic patch. Next, we will generalize the implementation to multiple patches and to patches containing EVs. According to Figure 14, a curved triangle $\Upsilon_{a}$ on the limit surface corresponds to the image of a straight-sided triangle $\Lambda_{a}$ in the parametric space, which is defined by the coordinates of the vertices, $S^{-1}\left(\mathbf{x}_{1}\right), S^{-1}\left(\mathbf{x}_{2}\right)$ and $S^{-1}\left(\mathbf{x}_{3}\right)$. As described in Section 3, these coordinates are stored during mesh generation.

In the NEFEM formulation, the shape functions are defined in the physical space and the numerical integration is performed with a special mapping from a properly defined parametric space to the curved elements. In particular, any curved tetrahedral element $\Omega_{a}$ with a face $\Upsilon_{a}$ on the boundary (see Figure 15), is described as an affine combination of $\Upsilon_{a}$ and the interior vertex $\mathbf{x}_{4}$. The mapping $\Psi$ to do so is defined as follows:

$$
\begin{array}{r}
\Psi: \Lambda_{a} \times[0,1] \rightarrow \Omega_{a} \\
(u, v, w) \mapsto \Psi(u, v, w):=(1-w) S(u, v)+w \mathbf{x}_{4} .
\end{array}
$$




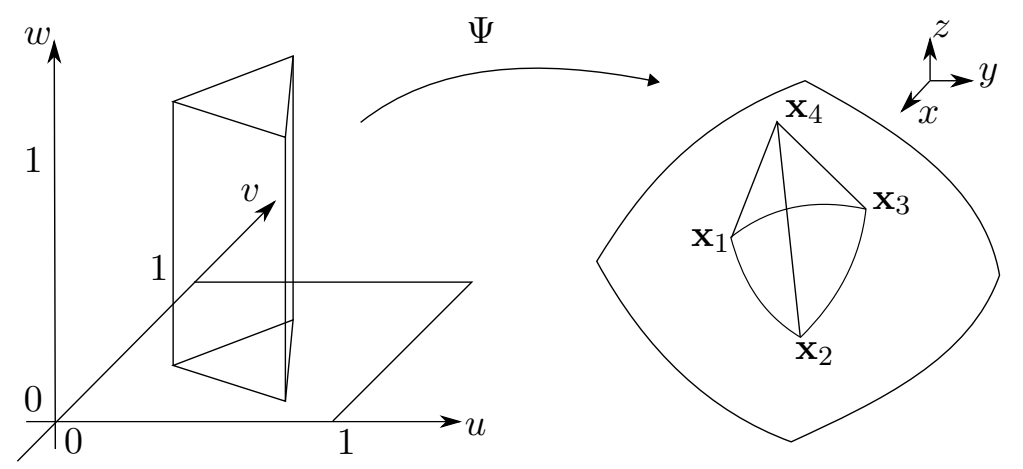

Figure 15: Mapping for a tetrahedral element with a curved face on the boundary. A transformation from a prism to the curved element $\Omega_{a}$ is defined.

Likewise, the elements with a curved edge on the boundary are parametrized using an affine combination of one of the two faces (sharing the edge) and the opposite vertex. For instance, in the case of the curved element in Figure 16 , the face $\Upsilon_{\mathbf{x}_{3}}$, which is opposite to $\mathbf{x}_{4}$, is parametrized as

$$
\begin{array}{r}
\Theta_{\mathbf{x}_{3}}:[0,1]^{2} \rightarrow \Upsilon_{\mathbf{x}_{3}} \\
(\varrho, \sigma) \mapsto \Theta_{\mathbf{x}_{3}}(\varrho, \sigma):=(1-\sigma) C(\varrho)+\sigma \mathbf{x}_{3},
\end{array}
$$

where $C(\varrho)$ parametrizes the curved edge $\Gamma_{a}$ by mapping the points on the segment from $S^{-1}\left(\mathbf{x}_{1}\right)$ to $S^{-1}\left(\mathbf{x}_{2}\right)$ in the parametric space to $\Gamma_{a}$, such that $\mathbf{x}_{1}=C(0)$ and $\mathbf{x}_{2}=C(1)$. Similarly, the face $\Upsilon_{\mathbf{x}_{4}}$, which is opposite to $\mathbf{x}_{3}$, is parametrized as

$$
\begin{array}{r}
\Theta_{\mathbf{x}_{4}}:[0,1]^{2} \rightarrow \Upsilon_{\mathbf{x}_{4}} \\
(\varrho, \tau) \mapsto \Theta_{\mathbf{x}_{4}}(\varrho, \tau):=(1-\tau) C(\varrho)+\tau \mathbf{x}_{4} .
\end{array}
$$

Once the parametrization for the faces is defined, a curved element can either be parametrized as

$$
\begin{array}{r}
\Phi_{\sigma \tau}[0,1]^{3} \rightarrow \Omega_{a} \\
(\varrho, \sigma, \tau) \mapsto \Phi_{\sigma \tau}(\varrho, \sigma, \tau):=(1-\tau) \Theta_{\mathbf{x}_{3}}(\varrho, \sigma)+\tau \mathbf{x}_{4}
\end{array}
$$

or as

$$
\begin{array}{r}
\Phi_{\tau \sigma}[0,1]^{3} \rightarrow \Omega_{a} \\
(\varrho, \sigma, \tau) \mapsto \Phi_{\tau \sigma}(\varrho, \sigma, \tau):=(1-\sigma) \Theta_{\mathbf{x}_{4}}(\varrho, \tau)+\sigma \mathbf{x}_{3},
\end{array}
$$

where the subscripts $\sigma \tau$ and $\tau \sigma$ indicate the order followed for the parametrization. Altogether, in the case of $\Phi_{\sigma \tau}$ an affine combination between $\Upsilon_{\mathbf{x}_{3}}$ and $\mathbf{x}_{4}$ is considered, while for $\Phi_{\tau \sigma}, \Upsilon_{\mathbf{x}_{4}}$ is combined with $\mathbf{x}_{3}$. 

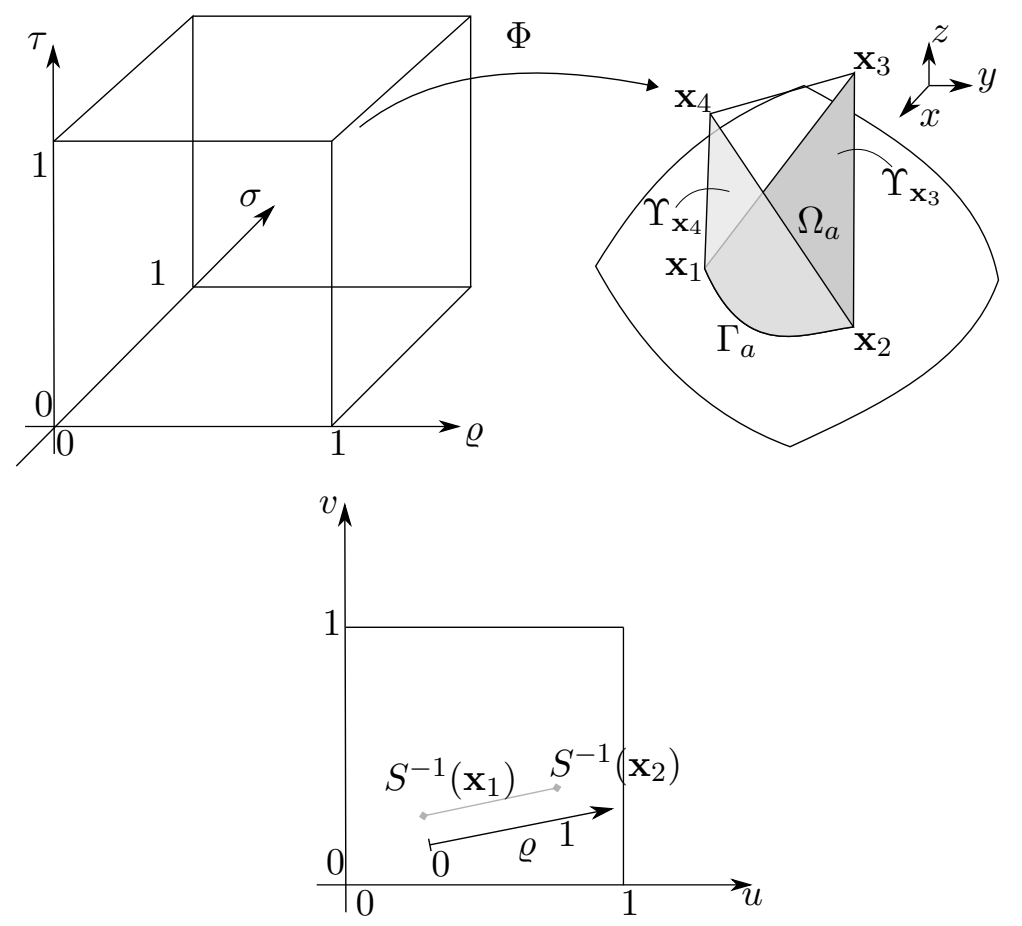

Figure 16: Mapping for a tetrahedral element with a curved edge on the boundary. A direction $\varrho$ is defined in the parametric space so that $S^{-1}\left(\mathbf{x}_{1}\right)$ corresponds to $\varrho=0$ and $S^{-1}\left(\mathbf{x}_{2}\right)$ to $\varrho=1$. Then a transformation from a cube to the curved element $\Omega_{a}$ is defined.

\subsection{Numerical integration}

As the shape functions are defined in the physical space, the parameterizations described in the previous section are used to compute surface and volume integrals on the curved elements. These integrals are required for the construction of the mass and stiffness matrices and to calculate the force vectors. For the sake of simplicity, in this work we will limit our attention only to linear elements, where the shape functions at a point $\boldsymbol{x}=\left[\begin{array}{lll}x & y & z\end{array}\right]$ are given by:

$$
\left\{\begin{array}{l}
\phi_{1}(\boldsymbol{x}) \\
\phi_{2}(\boldsymbol{x}) \\
\phi_{3}(\boldsymbol{x}) \\
\phi_{4}(\boldsymbol{x})
\end{array}\right\}=\left[\begin{array}{cccc}
x_{1} & x_{2} & x_{3} & x_{4} \\
y_{1} & y_{2} & y_{3} & y_{4} \\
z_{1} & z_{2} & z_{3} & z_{4} \\
1 & 1 & 1 & 1
\end{array}\right]^{-1}\left[\begin{array}{l}
x \\
y \\
z \\
1
\end{array}\right]
$$

For a discussion on higher order shape functions and p-refinement we refer to [7]. 
When natural boundary conditions are imposed, an integral of a function $f$ over a curved boundary face $\Upsilon_{a}$ is given by

$$
\int_{\Upsilon_{a}} f d A=\int_{\Lambda_{a}} f(S(u, v))\left\|J_{S(u, v)}\right\| d A
$$

where $J_{S(u, v)}=\frac{\partial S}{\partial u} \times \frac{\partial S}{\partial v}$ is the fundamental vector product of the surface. If the curved triangle $\Upsilon_{a}$ belongs completely to a single bicubic patch, the numerical integration is performed employing a Gauss quadrature over the parametric triangle - see e.g. [48]. As $\left\|J_{S(u, v)}\right\|$ is not polynomial, exact integration for a polynomial function $f$ is in general not possible. However, in practice the machine precision can be reached by increasing the number of Gauss points. For instance, if $f$ is constant, a $10^{\text {th }}$ order rule from [48] is usually sufficient. The situation changes when $\Upsilon_{a}$ belongs to multiple patches. In this case, the numerical quadrature has to be implemented in a way that considers the change of parametrization within each patch. The easiest way to obtain such an implementation is to divide the parametric triangle $\Lambda_{a}$ into subtriangles that belong to only one patch. Figure 17 illustrates an example in which a curved face $\Upsilon_{a}$ belongs to four different patches. The parametric triangle $\Lambda_{a}$ is split into seven subtriangles, whereupon Gauss quadrature can be applied to each one individually. Alternatively, Gauss quadratures for quadrilaterals could be implemented for the regions where the subtriangles form quadrilaterals. However, the total number of Gauss points would remain in the same order of magnitude.

In the implementation using subdivision surfaces, special care is required for those faces that belong to patches containing an EV. According to figure 8, the region surrounding an EV can be seen as an infinite sequence of spline rings. The respective patch can - in the parametric domain - be subdivided into an infinite sequence of virtual subpatches (Figure 7). Like before, the parametric triangle $\Lambda_{a}$ has to be split in order to take the different parametrization in each virtual subpatch into account. Figure 18 illustrates the case of a triangle in the neighbourhood of an EV.

The situation becomes more complicated for those triangles that have a vertex that corresponds to an EV. In this case, the parametric triangle $\Lambda_{a}$ should be split into an infinite number of subtriangles. From a numerical point of view this operation is obviously not feasible. Therefore, in practice, the number of virtual subdivisions has to be limited for the numerical quadrature. In Figure 19, 1, 2, 3, 4 and 5 virtual subdivision steps - as well as the relative triangulations - are shown for an element with a vertex that corresponds to an EV. By limiting the number of virtual subdivisions, an error is introduced in the numerical evaluation of eq. (17). However, machine 


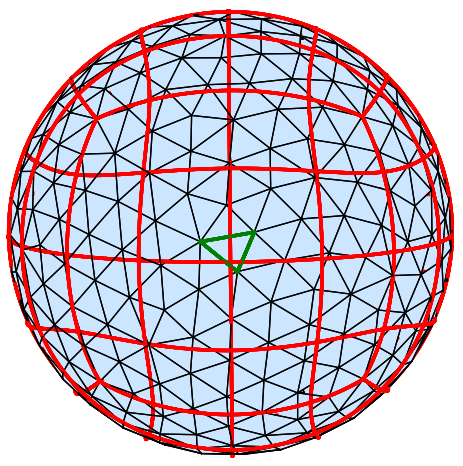

(a)

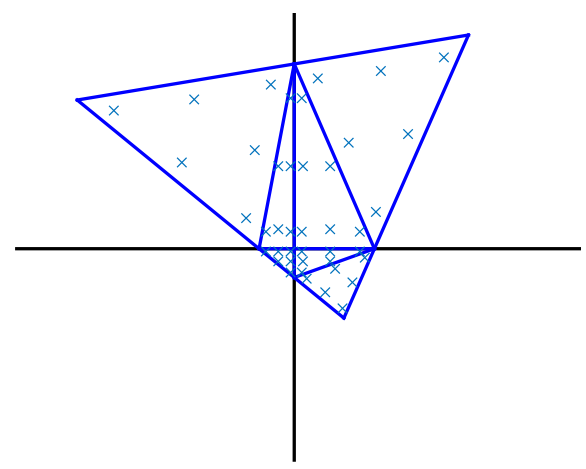

(b)

Figure 17: A curved face (outlined in dark green) intersecting four different regular patches (a) and the respective triangle in the parametric domain (b), which is split into subtriangles in order to take the change of parametrization into account. Gauss quadrature is applied in each subtriangle.

precision can still be reached with a finite number of subdivisions. This aspect will be further discussed in Section 5, where it will be shown that using a sufficient number of subdivision steps the elements are patch-test compliant up to machine precision.

Having a vertex of $\Upsilon_{a}$ corresponding to an EV is useful in the implementation because it facilitates the definition of the parametric triangles $\Lambda_{a}$. In fact, in the mesh generation process, the connectivity between the nodes is specified only in the physical space. If $\Upsilon_{a}$ belongs to only one patch, $\Lambda_{a}$ is easily obtained by connecting the three parametric points $S^{-1}\left(\mathbf{x}_{1}\right), S^{-1}\left(\mathbf{x}_{2}\right)$ and $S^{-1}\left(\mathbf{x}_{3}\right)$. The definition of $\Lambda_{a}$ is still straightforward in the case that the vertices belong to different patches (either to regular patches like in Figure 17 , or to patches containing EVs), but would become more complicated if the curved face $\Upsilon_{a}$ would contain an EV. In this case, some special rule has to be defined to connect the three points that correspond to the vertices in the parametric space. On the other hand, by constraining all EVs to nodes in the physical mesh, the EVs cannot be within a triangle. As a result, the definition of all parametric triangles $\Lambda_{a}$ is straightforward. For the same reasons, the condition $h(\mathbf{x})<H(\mathbf{x})$ also facilitates the definition of $\Lambda_{a}$ in the implementation.

While eq. (17) defines a surface integral that is used for the imposition of the natural boundary conditions, in the assembly of the mass and stiffness matrices volume integrals have to be evaluated. In the case of an element 


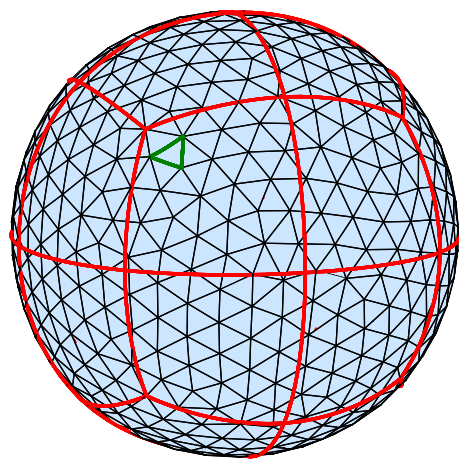

(a)

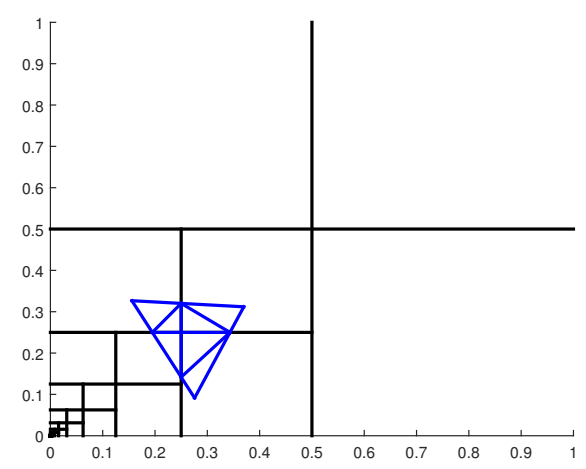

(b)

Figure 18: A curved face (outlined in dark green) in the neighbourhood of an $E V$ (a) and the respective triangle in the parametric domain (b), that is split into subtriangles in order to take the change of parametrization within the virtual subpatches into account. Gauss quadrature is applied in each subtriangle.

$\Omega_{a}$ with a curved face on the boundary, the volume integral of a function $f$ is calculated considering the parametrization given by (11) in the following way:

$$
\int_{\Omega_{a}} f d V=\int_{\Lambda_{a}} \int_{0}^{1} f(\Psi(u, v, w))\left|J_{\Psi(u, v, w)}\right| d V
$$

where $\left|J_{\Psi(u, v, w)}\right|$ is the determinant of the Jacobian of the transformation $\Psi$. On implementing numerical quadrature in $\Lambda_{a} \times[0,1]$ a tensor product is defined between a quadrature rule for the triangle in $\Lambda_{a}$ and a $1 \mathrm{D}$ Gauss-Legendre quadrature in $[0,1]$. Similar to the case of surface integrals, changes in parameterization have to be considered when $\Lambda_{a}$ belongs to different patches. However, due to the nature of the volume transformation, only the quadrature on $\Lambda_{a}$ has to be modified. The quadrature in the direction of $w$ remains unchanged.

It is important to remark that the parametrization from a prism to a curved tetrahedron given by (11) allows a significant reduction of the total number of Gauss points. In fact, $\left|J_{\Psi(u, v, w)}\right|$ is a quadratic function of the third parameter $w$ whereas $\Psi$ is linear with respect to $w$. Therefore, if the function $f$ is of polynomial degree $k$, exact integration is possible in the direction of $w$ by applying a Gauss-Legendre quadrature of order at least $k+2$. The decoupling of $w$ from the $u$ and $v$ directions is particularly important in the NEFEM framework, because $\left|J_{\Psi(u, v, w)}\right|$ is in general a 


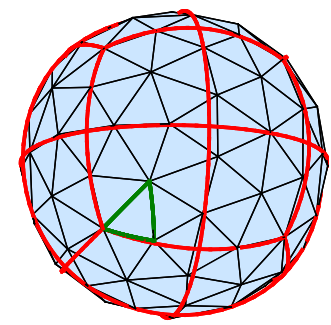

(a)

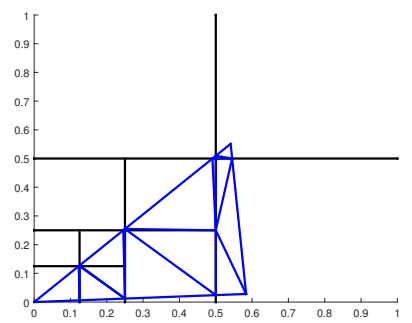

(d)

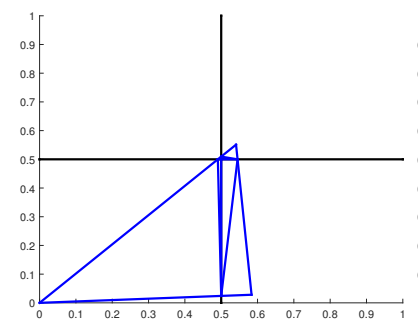

(b)

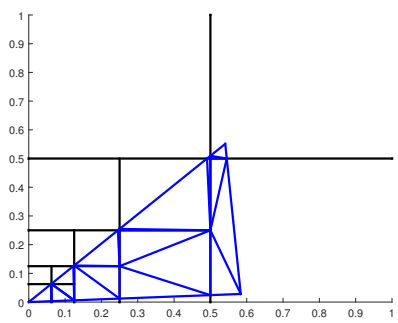

(e)

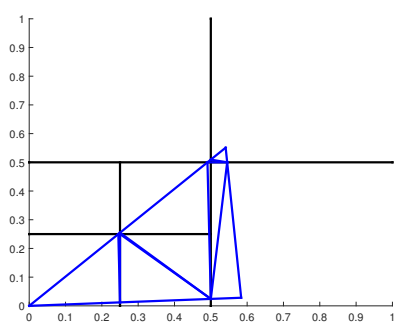

(c)

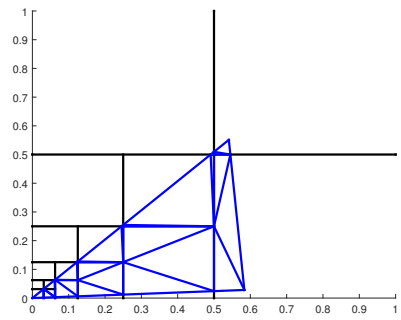

(f)

Figure 19: A curved face (outlined in dark green) with a vertex corresponding to an $E V(a)$ and the subtriangulations of the parametric triangle $\Lambda_{a}$ for 1, 2, 3, 4 and 5 virtual subdivisions.

rational function of $u$ and $v$ which means that exact integration is, in general, not feasible for these directions. However, by decoupling $w$, the evaluation of integral (18) has the same complexity as an integral over a trimmed NURBS surface corresponding to $\Upsilon_{a}$.

In the case of subdivision surfaces, $\left|J_{\Psi(u, v, w)}\right|$ is polynomial with respect to $u$ and $v$, the highest term containing $u^{8} v^{8}$. Therefore, for a function $f$ of degree $k$, the term $u^{3 k+8} v^{3 k+8}$ will be present in the integrand. While exact integration is still possible, in practice machine precision can be reached with a significantly smaller number of Gauss points. For instance, in the case of a constant function $(k=0)$, a $10^{\text {th }}$ order rule on $\Lambda_{a}$, combined with a quadratic rule in the direction of $w$, is sufficient to reach machine precision in most cases. In addition, as it is shown in Section 5, a much coarser quadrature is enough to ensure the correct convergence of the method.

We also note that if a transformation from a parametric tetrahedron to the curved element would have been used, a $10^{t h}$ order rule in such tetrahedron would require many more Gauss points compared to the case where $\Lambda_{a}$ is decoupled from the $w$ direction. 
In the case of an element $\Omega_{a}$ with a curved edge on the boundary, the volume integral of a function $f$ can be calculated considering parameterizations (14) or (15) in the following way:

$$
\int_{\Omega_{a}} f d V=\int_{0}^{1} \int_{0}^{1} \int_{0}^{1} f(\Phi(\varrho, \sigma, \tau))\left|J_{\Phi(\varrho, \sigma, \tau)}\right| d V
$$

where $\left|J_{\Phi(\varrho, \sigma, \tau)}\right|$ is the determinant of the Jacobian of the transformation $\Phi$. On implementing numerical quadrature, a tensor product between 1D Gauss-Legendre rules in the 3 directions $\varrho, \sigma$ and $\tau$ is defined. In this case the transformation $\Phi$ is linear with respect to $\sigma$ and $\tau$, whereas $\left|J_{\Phi(\varrho, \sigma, \tau)}\right|$ is quadratic with respect to this parameters. Therefore, the complexity of the problem is decoupled for both $\sigma$ and $\tau$ from the $\varrho$ direction. Again, while in theory an exact integration would be possible also with respect to $\varrho$, the machine precision is normally reached using a smaller number of Gauss points. In particular, for a constant function $f$, seven points (defining a $13^{\text {th }}$ order rule) are usually sufficient. Just as for the case of curved faces, composite rules should be considered in the direction of $\varrho$ when a change of parametrization occurs. This can be realized by splitting the segment that connects $S^{-1}\left(\mathbf{x}_{1}\right)$ and $S^{-1}\left(\mathbf{x}_{2}\right)$.

As far as the computational times are concerned, the matrix assembling procedure is expected to be more expensive with respect to a standard FEM code because the aforementioned integration approach requires a higher number of shape function evaluations and, in addition, it is not possible to make use of caching operations as in isoparametric FEMs. However, we note that the total number of elements on the boundary is proportional to $h^{-2}$ while the total number of elements is proportional to $h^{-3}$. Therefore, for large scale problems, the time required to process the elements on the boundary becomes asymptotically smaller than the time required for the interior elements. A practical example on this aspect is given in Section 5 . It is also worth to remark that the number of elements with a node corresponding to an EV, where the special refinement strategy is required, is very small if compared to the total number of elements because the number of EVs is a feature of the subdivision model and remains constant when the FEM mesh is refined.

\section{Numerical examples}

The validity of the method is demonstrated by several numerical examples, including a convergence analysis for Laplace problems, an acoustic 


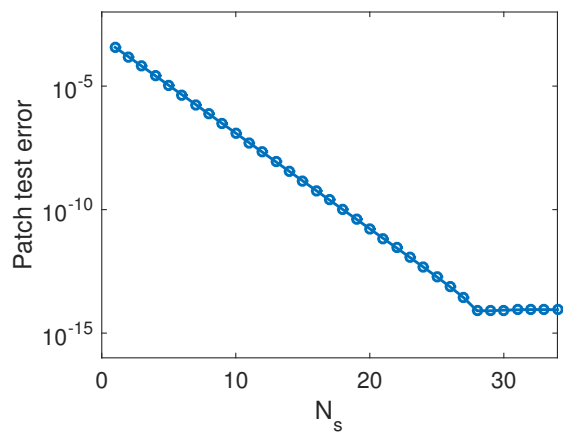

Figure 20: Patch test error as a function of the number of virtual subdivisions $N_{s}$.

Helmholtz problem and the computation of the eigenmodes associated with the Helmholtz equation on a rocker arm Catmull-Clark model.

\subsection{Laplace problems}

In this section the following boundary value problem (BVP) for the Laplace equation is considered:

$$
\begin{cases}\Delta u=0 & \text { in } \Omega \\ \nabla u \cdot \boldsymbol{n}=g_{n} & \text { on } \partial \Omega,\end{cases}
$$

where $\boldsymbol{n}$ is the outward unit normal vector to $\partial \Omega$ and $g_{n}$ is the normal flux. For the problem to be well posed, $g_{n}$ is chosen such that it verifies the compatibility condition

$$
\oint_{\partial \Omega} g_{n} d A=0
$$

First, we follow the approach employed in [49] in order to assess the validity of the integration procedure around EVs by mean of the patch test. In particular, the linear field $u(\boldsymbol{x})=x+y+z$ is reproduced in the element in Figure 19 by imposing the analytical flux $\nabla u=[1,1,1]$ and a Dirichlet condition on the interior node $\mathbf{x}_{4}$.

As discussed in the previous section, an accurate quadrature with a $10^{\text {th }}$ order rule in the parametric triangles is considered and, therefore, the only source of error is the integration error around the EV.

In Figure 20 we can observe how the error (defined as the mean error on the solution at $\mathbf{x}_{1}, \mathbf{x}_{2}$ and $\mathbf{x}_{3}$ ) decreases when a higher number of virtual subdivisions is considered, until reaching machine precision. These results are in agreement with Scott's thesis [49], where it is observed that the patch 


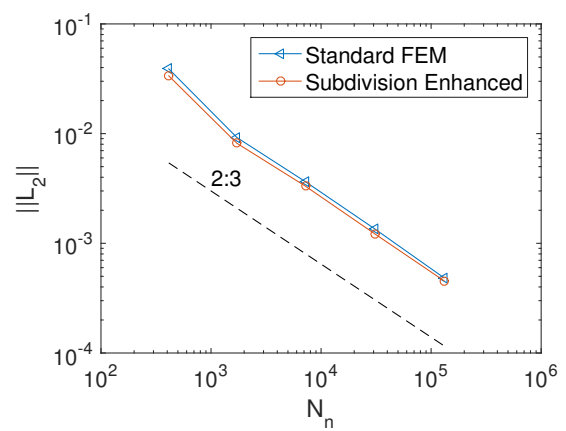

(a)

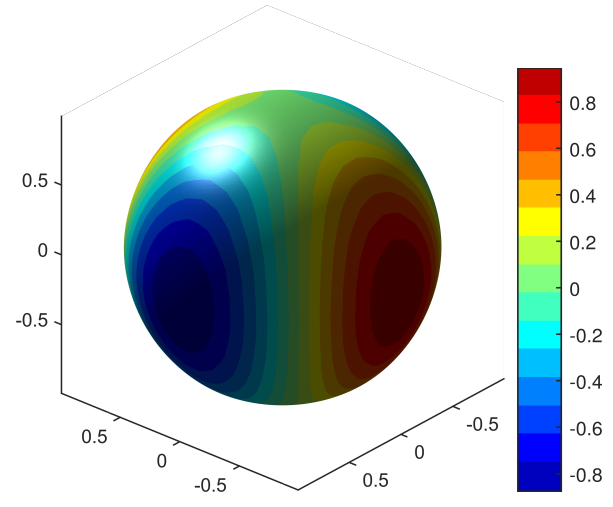

(b)

Figure 21: Convergence of the $L_{2}$ norm of the error for the Laplace problem on a spherical-like domain (a) and the solution approximating $u(\boldsymbol{x})=x^{2}-y^{2}$ for a mesh with 3000 nodes (b).

test error can reach machine precision after about 25 virtual subdivisions. A similar behavior for IgA with subdivision surfaces is also reported in [28] and [50].

The problem stated in (20) is then solved for three subdivision models, viz. an approximation of a sphere, the model in Figure 1 and an A-shaped model. The approximation of a spherical surface with the Catmull-Clark scheme is discussed in Section 5.2.

The quadratic field $u(\boldsymbol{x})=x^{2}-y^{2}$ is approximated by imposing the analytical normal flux $\nabla u=[2 x,-2 y, 0]$ on $\partial \Omega$ and a Dirichlet condition with the exact value of $u$ at a single point on the boundary. In Figure 21 the convergence of the $L_{2}$ norm of the error is considered for the approximation of the sphere. The asymptotic rate of convergence is consistent with the theory [51]. A plot of the solution on the boundary for a mesh with 3000 nodes is also included. On comparing the method with standard finite elements, a slight improvement of the accuracy is observed. This is the result of directly imposing the boundary conditions on the curved domain.

As far as the numerical integration is concerned, the same accurate integration strategy of the patch-test is considered for the enhanced elements and 4 virtual subdivision steps are applied around the EVs. Since the global convergence is studied here, this is enough to obtain the correct convergence behaviour and no improvement is observed for more virtual subdivisions. On the other hand, the convergence curve remains visually the same even if no 


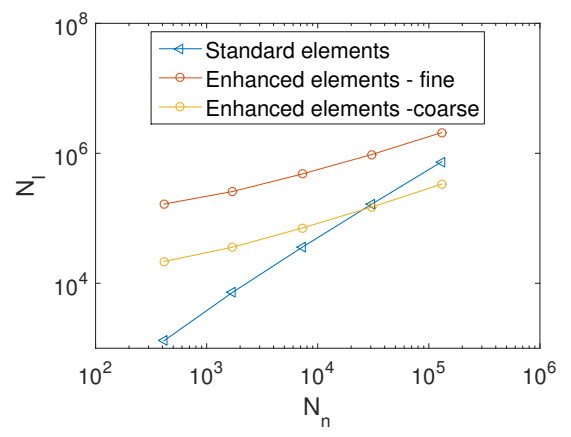

Figure 22: Number of integration points $N_{I}$ in the standard and enhanced elements for the Laplace problem on a spherical-like domain.

virtual subdivisions are applied. At the same time, if a coarser quadrature is used in the enhanced elements, no apparent change is observed in the convergence plot. For this particular application, such a result was confirmed up to the case of an integration strategy with a $2^{\text {nd }}$ order rule on $\Lambda_{a}$ for the enhanced elements with a face on the boundary and a Gauss-Legendre rule with 2 points for the elements with an edge on the boundary.

In order to compare the computational effort needed for the enhanced elements to that needed for the standard ones, in Figure 22 the number of integration points is plotted. In the case of standard elements, since linear shape functions are used, this is equivalent to consider one integration point per element. If a fine integration is considered, it can be observed how, for small meshes, most of the computational effort is due to the integration of the enhanced elements. However, as discussed in Section 4, the complexity of such a procedure becomes asymptotically smaller with respect to the standard elements. The situation improves significantly when the coarser integration mentioned above is considered. In this case, the two curves intersect for a number of nodes of approximately 30.000 and, therefore, for a higher number of nodes most of the computational effort is consumed by the standard elements in the interior of the mesh.

The model from Figure 1 and an A-shaped model are considered respectively in Figures 23 and 24. The correct asymptotic rates of convergence and a slight improvement with respect to standard finite elements are confirmed also for these examples. 


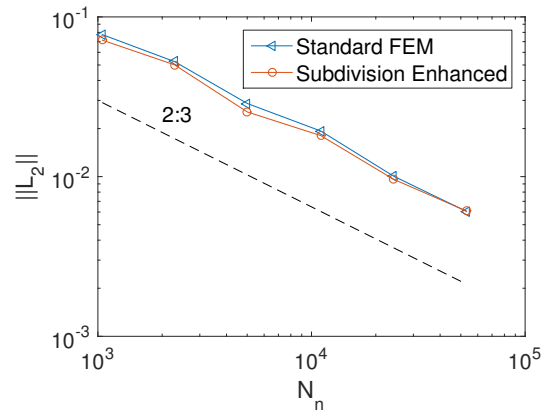

(a)

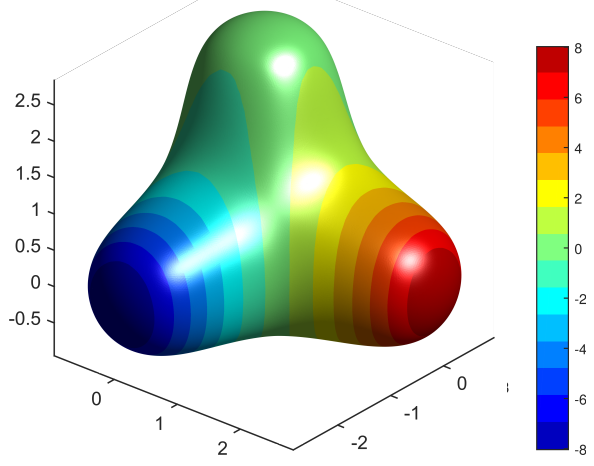

(b)

Figure 23: Convergence of the $L_{2}$ norm of the error for the Laplace problem on the model from Figure 1 (a) and the solution approximating $u(\boldsymbol{x})=$ $x^{2}-y^{2}$ for a mesh with 5600 nodes (b).

\subsection{Helmholtz problems}

5.2.1. Acoustic field inside a sphere with vibrating walls

In this section we consider the following Helmholtz problem:

$$
\begin{cases}\Delta p+k^{2} p=0 & \text { in } \Omega \\ \frac{\mathrm{j}}{\rho_{0} \omega} \nabla p \cdot \boldsymbol{n}=\bar{v}_{n} & \text { on } \partial \Omega,\end{cases}
$$

that describes the acoustic field generated within a sphere, whose walls are vibrating with a normal velocity $\bar{v}_{n}$ at the angular frequency $\omega$. In the aforementioned system, $\rho_{0}$ is the density of the fluid medium inside the sphere and $k$ is the wavenumber, related to the frequency by the following relation:

$$
\omega=\frac{k}{c}
$$

where $c$ is the speed of sound.

Considering the radial symmetry and the expression of the Helmholtz equation in spherical coordinates[52], Problem (22) can be expressed as

$$
\begin{cases}\frac{1}{r^{2}} \frac{\partial}{\partial r}\left(r^{2} \frac{\partial p(r)}{\partial r}\right)+k^{2} p(r)=0 & r \in\left[\begin{array}{ll}
0 & R
\end{array}\right], \\
\frac{\mathrm{j}}{\rho_{0} \omega} \frac{\partial p(r)}{\partial r}=\bar{v}_{n} & r=R,\end{cases}
$$




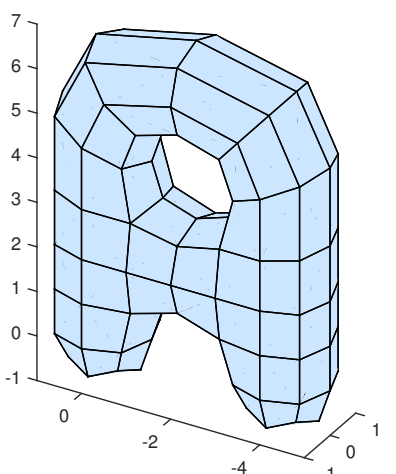

(a)

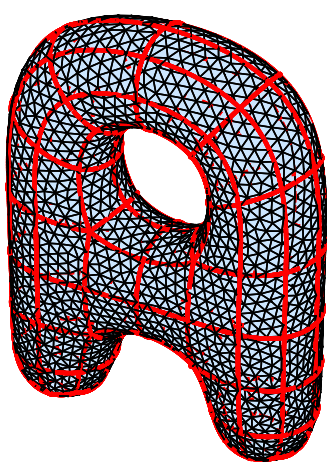

(b)

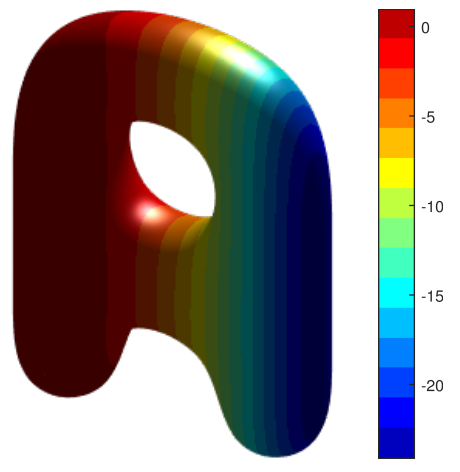

(c)

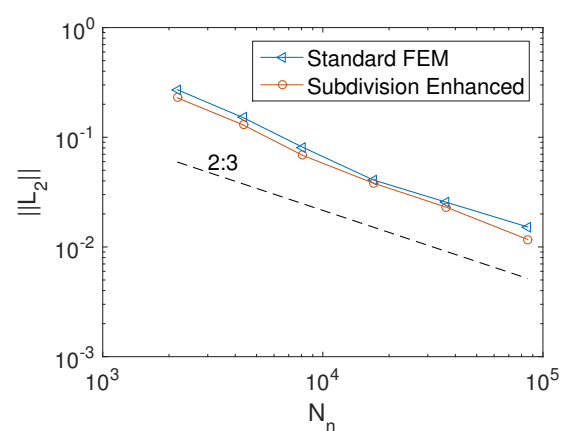

(d)

Figure 24: The Laplace problem on an A-shaped model; the original Catmull-Clark control net (a); a mesh with roughly 15000 nodes (b) and the respective solution approximating $u(\boldsymbol{x})=x^{2}-y^{2}(c)$; the convergence of the $L_{2}$ norm of the error (d). 
whose solution is given by

$$
p(r)=-\mathrm{j} \bar{v}_{n} \rho_{0} \omega \frac{e^{-\sqrt{-k^{2}}(r-1)}\left(e^{2 \sqrt{-k^{2}} r}-1\right)}{\left(e^{2 \sqrt{-k^{2}}}\left(\sqrt{-k^{2}}-1\right)+\sqrt{-k^{2}}+1\right) r} .
$$

The problem is solved considering the following parameters: $\rho_{0}=1.21 \mathrm{~kg} / \mathrm{m}^{3}$, $\omega=215 \mathrm{rad} / \mathrm{s}, c=343 \mathrm{~m} / \mathrm{s}$ and the analytical solution is used as reference for the convergence study.

As mentioned earlier, the Catmull-Clark subdivision scheme is not able to exactly represent a sphere, but a good approximation can be found by minimizing the error in a least-square sense. In particular, the following approach is used in this work. Consider a sphere of unit radius centered at the origin. For a given point $\boldsymbol{x}^{h}$ that lies on the surface approximating the sphere, its projection on the exact surface $\boldsymbol{x}_{s}$ can be computed as

$$
\boldsymbol{x}_{s}=\frac{\boldsymbol{x}^{h}}{\left\|\boldsymbol{x}^{h}\right\|} .
$$

We define the error on the surface approximation as

$$
\varepsilon=\sqrt{\oint\left\|\boldsymbol{x}^{h}-\boldsymbol{x}_{s}\right\|^{2} d S} .
$$

and then we look for a subdivision model whose control net is chosen in a way that the aforementioned error is minimized. To this end, we start with a subdivision mesh of a cube, with some level of refinement $\mathcal{M}_{i}$. For each patch $\mathcal{S}_{i}^{k}(u, v)$, the points on the limit surface are given by:

$$
\boldsymbol{x}^{h}(u, v)=\boldsymbol{N}^{T}(u, v) \boldsymbol{P}
$$

where $\boldsymbol{N}^{T}(u, v)$ are the basis functions and $\boldsymbol{P}$ are the control points obtained from $\mathcal{M}_{i}$. On combining this relation with the definition of the error, the new control points $\boldsymbol{P}_{s}$ that better approximate the surface can be found by minimizing

$$
\min \sum_{k} \int_{0}^{1} \int_{0}^{1}\left\|\boldsymbol{N}^{T}(u, v) \boldsymbol{P}_{s}-\boldsymbol{x}_{s}(u, v)\right\|^{2} d u d v
$$

which leads to the following linear system:

$$
\boldsymbol{A P} \boldsymbol{P}_{s}=\boldsymbol{b}
$$




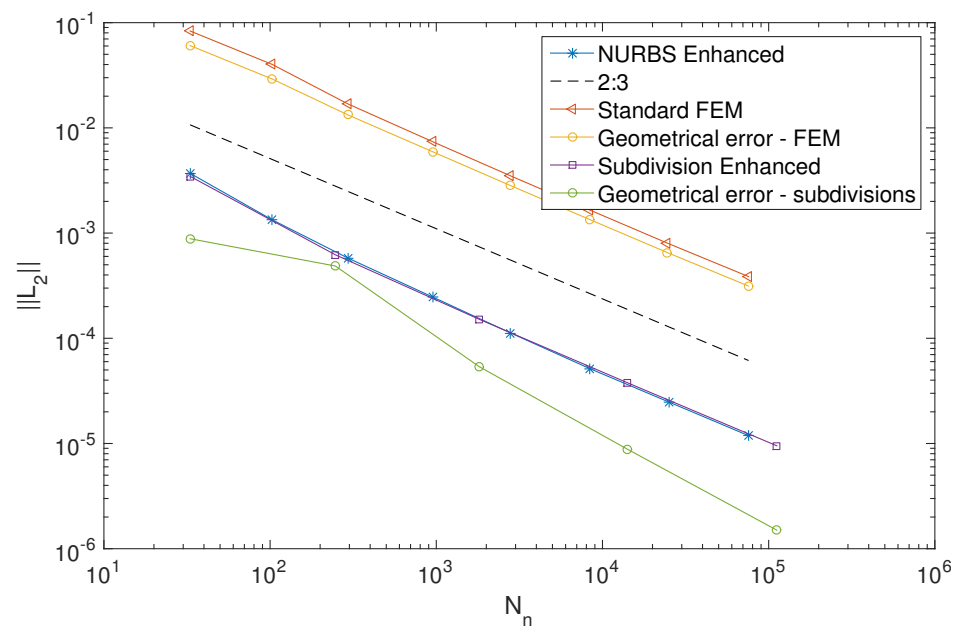

Figure 25: Convergence of the $L_{2}$ norm of the error as a function of the number of nodes $N_{n}$ for the Helmholtz problem on a spherical domain and geometrical error for the Catmull-Clark and the FEM approximations.

where

$$
\begin{gathered}
\boldsymbol{A}=\sum_{k} \int_{0}^{1} \int_{0}^{1} \boldsymbol{N}(u, v) \boldsymbol{N}^{T}(u, v) d u d v \\
\boldsymbol{b}=\sum_{k} \int_{0}^{1} \int_{0}^{1} \boldsymbol{N}(u, v) \boldsymbol{x}_{s}(u, v) d u d v .
\end{gathered}
$$

On solving System (30) the positions of the control points that best approximates the sphere are found.

Since the accuracy of this procedure depends on the number of points on $\mathcal{M}_{i}$, the control net is also refined during the convergence analysis, reported in Figure 25. In particular, each point in the convergence curve is calculated by starting from a different control net $\mathcal{M}_{i}$, adapting the control points in order to best approximate the sphere and then computing the FEM mesh with the algorithm described in Section 3.

We can observe how, in this case, a significant improvement is obtained respect to the standard FEM solution. In fact, it is well known in the NEFEM literature that the correct geometric representation is more important for wave problems rather than elliptic problems, since pollution errors can destroy the solution within the domain [6].

In the figure it is reported also the geometrical error defined in Eq. (27) for both the standard FEM and the subdivision enhanced models. We can 
observe how the error in the standard FEM solution is very close to the corresponding geometrical error, which limits the accuracy of the numerical solution. On the other hand, the geometrical error is lower than the error on the solution for the subdivision enhanced FEM.

To further confirm that the approximation introduced by the subdivision approach is not affecting the numerical solution, the problem is simulated also with NEFEM. In this case, the approach described in [53] is employed to tile the sphere with fourth-order Bézier patches and, in order to have a fair comparison, the force equilibrium method described in Section 3 is employed for the mesh generation.

We can observe how the NEFEM and the subdivision curves are practically matching, which confirms that the error in the field approximation is predominant respect to the geometrical error for subdivisions. Since they do not present any geometrical error, the meshes from the NEFEM simulation were used also for the standard FEM analysis.

It is worth to remark that, for higher order polynomial approximations, the FEM solution should converge with a higher rate than the error in the surface approximation, and therefore this would become a limitation of the method in spherical domains. However, this application is only an example to illustrate the potential of the subdivision enhanced approach, implemented with the Catmull-Clark scheme. NURBS-compatible subdivision schemes [33] would allow for an exact representation of the sphere, and therefore avoid the geometrical error.

\subsubsection{Rocker arm}

In order to consider a practical engineering application, the Helmholtz problem (which can be used to model thermal diffusion problems in the frequency domain) is studied on the rocker arm model in Figure 26.

The purpose of this example is only to prove the applicability of the method for a more topologically complex model. The computation of the first 3 non-zero eigenmodes for problem (22) is considered with both standard and subdivision enhanced elements. A mesh with roughly 150.000 nodes is employed.

Looking at Figure 26, it can be observed how, despite the high number of nodes, the geometrical approximation is still evident in the standard FEM solution. On the other hand, a smooth field over the exact geometry is obtained with the subdivision enhanced elements. 


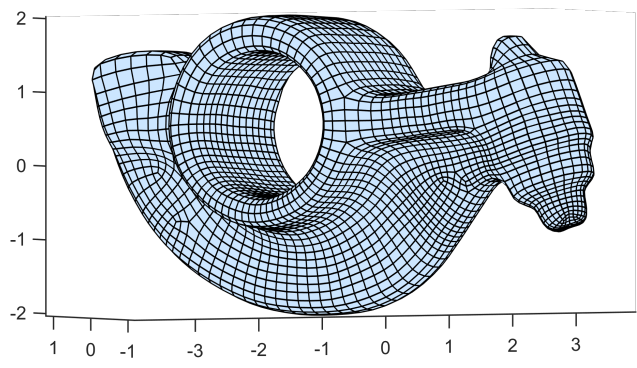

(a)

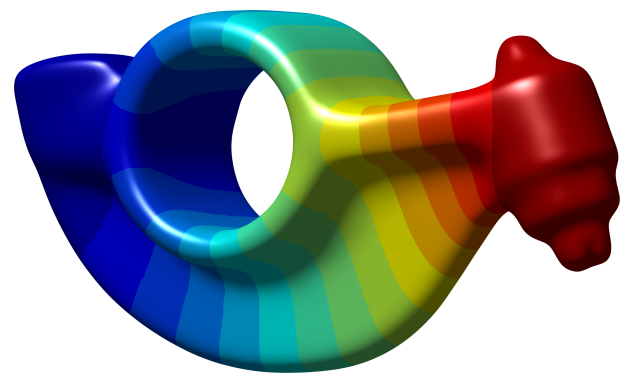

(c)

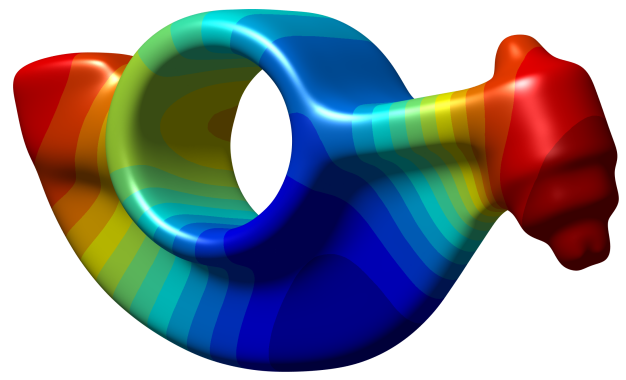

(e)

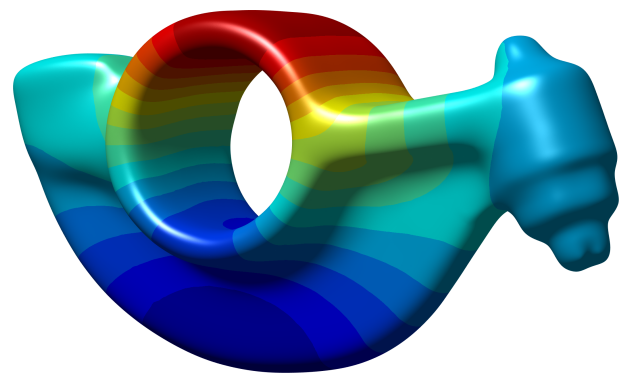

$(\mathrm{g})$

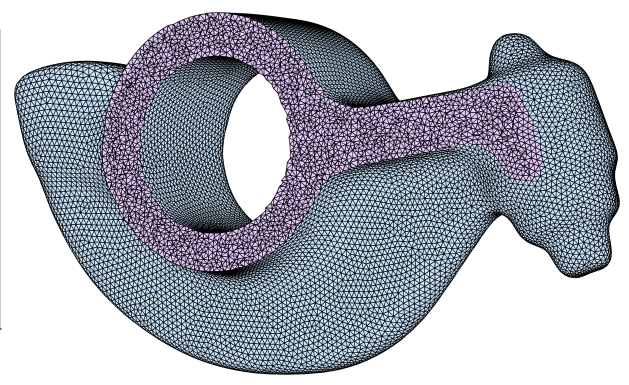

(b)

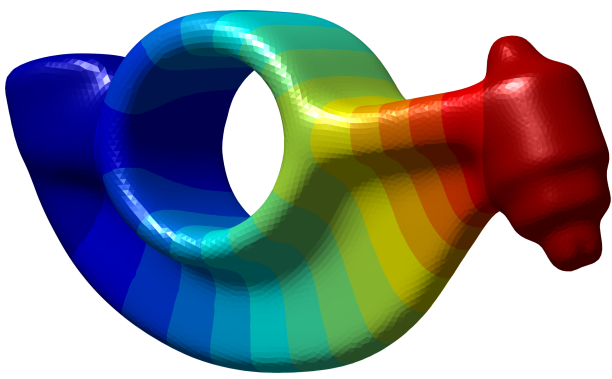

(d)

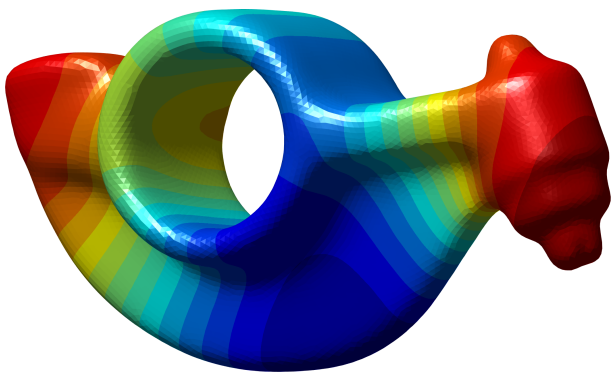

(f)

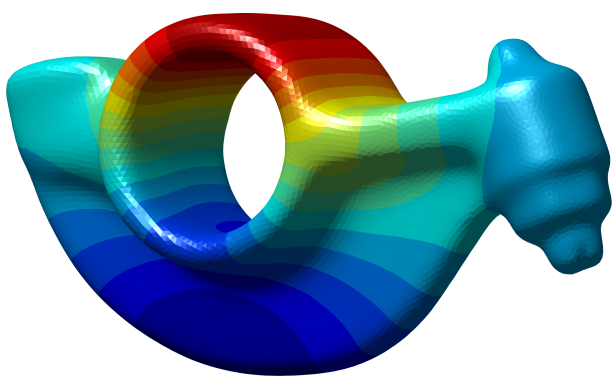

(h)

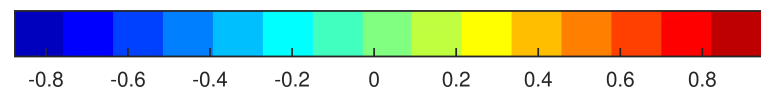

Figure 26: Helmholtz problem on $a_{34}$ ocker arm model; the Catmull-Clark control net (a), the FEM mesh with roughly 150000 nodes (b) and a comparison of the first 3 eigenmodes computed with subdivision enhanced FEM $(c-e-g)$ and standard FEM (d-f-h). 


\section{Concluding remarks}

In this work the concept of 3D NURBS Enhanced Finite Elements has been extended to volumes bounded by Catmull-Clark subdivision surfaces, resulting in a design-through-analysis methodology.

The mathematical formulation of NEFEM is directly applied to bicubic patches, while special integration rules are implemented to take the change of parametrization around special vertices into account. In particular, exact integration is possible for all triangles that do not intersect the EVs, while for the triangles with a vertex in correspondence with an EV, a virtual subdivision approach is used for the integration. This method has been validated using the patch test, where machine precision can be reached using a considerable number of subdivisions. However, in practice, a few subdivisions are sufficient to obtain engineering accuracy.

The main advantage of using the subdivision surface boundary representation is that the process of mesh generation is facilitated by the watertightness of the surface, especially if compared to models based on NURBS. At the same time, the exact geometry representation is considered in the numerical analysis with the NEFEM approach. As a result, a better integration between $\mathrm{CAD}$ and Finite Element analysis is obtained.

Catmull-Clark surfaces have been used for presenting the methodology as it is the most widely-used subdivision scheme. However, the extension to alternative schemes is expected to be straightforward and can be the topic of future research. This includes extensions to the Catmull-Clark scheme [32] as well as the NURBS-compatible scheme [33], which can both represent objects with sharp geometric features.

\section{Acknowledgments}

The Research Fund KU Leuven is gratefully acknowledged for its support. The research of P. Barendrecht has been funded within the European ITN Project BATWOMAN (No. 605867), under the FP7 Marie Curie Programme. The research of L. Coox is funded by a grant from the IWT Flanders. This work is also partially supported by Flanders Make, the strategic research center for the manufacturing industry.

\section{References}

[1] D. F. Rogers, An introduction to NURBS: with historical perspective. Elsevier, 2000. 
[2] T. J. Hughes, J. A. Cottrell, and Y. Bazilevs, "Isogeometric analysis: CAD, finite elements, NURBS, exact geometry and mesh refinement," Computer methods in applied mechanics and engineering, vol. 194, no. 39, pp. 4135-4195, 2005.

[3] J. A. Cottrell, T. J. Hughes, and Y. Bazilevs, Isogeometric analysis: toward integration of CAD and FEA. John Wiley \& Sons, 2009.

[4] R. Sevilla, S. Fernández-Méndez, and A. Huerta, "NURBS-enhanced finite element method (NEFEM)," Archives of Computational Methods in Engineering, vol. 18, no. 4, pp. 441-484, 2011.

[5] R. Sevilla, NURBS-enhanced finite element method (NEFEM). $\mathrm{PhD}$ thesis, 2009.

[6] R. Sevilla, S. Fernández-Méndez, and A. Huerta, "NURBS-enhanced finite element method (NEFEM)," International Journal for Numerical Methods in Engineering, vol. 76, no. 1, pp. 56-83, 2008.

[7] R. Sevilla, S. Fernández-Méndez, and A. Huerta, "3D NURBSenhanced finite element method (NEFEM)," International Journal for Numerical Methods in Engineering, vol. 88, no. 2, pp. 103-125, 2011.

[8] J. Warren and H. Weimer, Subdivision methods for geometric design: A constructive approach. Morgan Kaufmann, 2001.

[9] T. J. Cashman, "Beyond Catmull-Clark? A survey of advances in subdivision surface methods," in Computer Graphics Forum, vol. 31, pp. 42-61, Wiley Online Library, 2012.

[10] J. Stam, "Exact evaluation of Catmull-Clark subdivision surfaces at arbitrary parameter values," in Proceedings of the 25th annual conference on Computer graphics and interactive techniques, pp. 395-404, ACM, 1998.

[11] F. Cirak, M. Ortiz, and P. Schröder, "Subdivision surfaces: a new paradigm for thin-shell finite-element analysis," International Journal for Numerical Methods in Engineering, vol. 47, no. 12, pp. 2039-2072, 2000 .

[12] E. Grinspun, P. Krysl, and P. Schröder, "CHARMS: a simple framework for adaptive simulation," ACM transactions on graphics (TOG), vol. 21, no. 3, pp. 281-290, 2002. 
[13] D. Burkhart, B. Hamann, and G. Umlauf, "Isogeometric finite element analysis based on Catmull-Clark subdivision solids," in Computer Graphics Forum, vol. 29, pp. 1575-1584, Wiley Online Library, 2010.

[14] U. Zore, B. Jüttler, and J. Kosinka, "On the linear independence of truncated hierarchical generating systems," Journal of Computational and Applied Mathematics, vol. 306, pp. 200-216, 2016.

[15] A. Bakenov, "T-Splines : tensor product B-spline surfaces with Tjunctions," Master's thesis, Brigham Young University, 2001.

[16] T. W. Sederberg, J. Zheng, A. Bakenov, and A. Nasri, "T-splines and TNURCCs," in ACM transactions on graphics (TOG), vol. 22, pp. 477484, ACM, 2003.

[17] T. W. Sederberg, J. Zheng, D. Sewell, and M. Sabin, "Non-uniform recursive subdivision surfaces," in Proceedings of the 25th annual conference on Computer graphics and interactive techniques, pp. 387-394, ACM, 1998.

[18] M. Scott, R. Simpson, J. Evans, S. Lipton, S. Bordas, T. Hughes, and T. Sederberg, "Isogeometric boundary element analysis using unstructured T-splines ," Computer Methods in Applied Mechanics and Engineering, vol. 254, pp. 197 - 221, 2013.

[19] Y. Bazilevs, V. Calo, J. Cottrell, J. Evans, T. Hughes, S. Lipton, M. Scott, and T. Sederberg, "Isogeometric analysis using T-Splines," Computer Methods in Applied Mechanics and Engineering, vol. 199, no. 5-8, pp. 229-263, 2010.

[20] Y. Zhang, W. Wang, and T. J. Hughes, "Solid T-spline construction from boundary representations for genus-zero geometry," Computer Methods in Applied Mechanics and Engineering, vol. 249, pp. 185-197, 2012.

[21] X. Li, J. Zheng, T. W. Sederberg, T. J. Hughes, and M. A. Scott, "On linear independence of T-spline blending functions," Computer Aided Geometric Design, vol. 29, no. 1, pp. 63-76, 2012.

[22] G. Barequet, C. Duncan, S. Kumar, et al., "Rsvp: A geometric toolkit for controlled repair of solid models," Visualization and Computer Graphics, IEEE Transactions on, vol. 4, no. 2, pp. 162-177, 1998. 
[23] A. C. Sans and E. O. I. de Navarra, Robust volume mesh generation for non-watertight geometries. $\mathrm{PhD}$ thesis, $\mathrm{Ph}$. D. thesis) CIMNE, Barcelona (May 2014), 2014.

[24] A. Wawrzinek, K. Hildebrandt, and K. Polthier, "Koiter's thin shells on catmull-clark limit surfaces," in VMV, pp. 113-120, 2011.

[25] P. J. Barendrecht, "Isogeometric analysis for subdivision surfaces," Master's thesis, Eindhoven University of Technology, 2013.

[26] T. Nguyen, K. Karčiauskas, and J. Peters, "A comparative study of several classical, discrete differential and isogeometric methods for solving Poissons equation on the disk," Axioms, vol. 3, no. 2, pp. 280-299, 2014.

[27] A. Riffnaller-Schiefer, U. H. Augsdörfer, and D. W. Fellner, "Isogeometric Analysis for Modelling and Design," in EG 2015 - Short Papers (B. Bickel and T. Ritschel, eds.), The Eurographics Association, 2015.

[28] B. Jüttler, A. Mantzaflaris, R. Perl, and M. Rumpf, "On isogeometric subdivision methods for PDEs on surfaces," arXiv preprint arXiv:1503.03730, 2015.

[29] A. Wawrzinek and K. Polthier, "Integration of generalized B-spline functions on Catmull-Clark surfaces at singularities," Computer-Aided Design, 2016.

[30] E. Catmull and J. Clark, "Recursively generated B-spline surfaces on arbitrary topological meshes," Computer-Aided Design, vol. 10, no. 6, pp. $350-355,1978$.

[31] J. Peters and U. Reif, Subdivision surfaces. Springer, 2008.

[32] T. DeRose, M. Kass, and T. Truong, "Subdivision surfaces in character animation," in Proceedings of the 25th annual conference on Computer graphics and interactive techniques, pp. 85-94, ACM, 1998.

[33] T. J. Cashman, NURBS-compatible subdivision surfaces. PhD thesis, Cashman, Thomas J., 2010.

[34] J. Shen, J. Kosinka, M. A. Sabin, and N. A. Dodgson, "Conversion of trimmed NURBS surfaces to Catmull-Clark subdivision surfaces," Computer Aided Geometric Design, vol. 31, no. 7, pp. 486-498, 2014. 
[35] M. Antonelli, C. V. Beccari, G. Casciola, R. Ciarloni, and S. Morigi, "Subdivision surfaces integrated in a cad system," Computer-Aided Design, vol. 45, no. 11, pp. 1294-1305, 2013.

[36] D. Burkhart, Subdivision for Volumetric Finite Elements. PhD thesis, University of Kaiserslautern, 2011.

[37] N. A. Dodgson, U. H. Augsdörfer, T. J. Cashman, and M. A. Sabin, "Deriving box-spline subdivision schemes," in Mathematics of Surfaces XIII, pp. 106-123, Springer, 2009.

[38] H. Prautzsch, W. Boehm, and M. Paluszny, Bézier and B-spline techniques. Springer Science \& Business Media, 2002.

[39] M. A. Sabin, Analysis and Design of Univariate Subdivision Schemes. Springer, 2010.

[40] D. Doo and M. Sabin, "Behaviour of recursive division surfaces near extraordinary points," Computer-Aided Design, vol. 10, no. 6, pp. 356360, 1978.

[41] U. H. Augsdörfer, N. A. Dodgson, and M. A. Sabin, "Tuning subdivision by minimising gaussian curvature variation near extraordinary vertices," in Computer Graphics Forum, vol. 25, pp. 263-272, Wiley Online Library, 2006.

[42] L.-E. Andersson and N. F. Stewart, Introduction to the mathematics of subdivision surfaces. SIAM, 2010.

[43] D. S. Lo, Finite Element Mesh Generation. CRC Press, 2014.

[44] P.-O. Persson, Mesh generation for implicit geometries. PhD thesis, 2004.

[45] P. Persson and G. Strang, "A simple mesh generator in matlab," SIAM Review, vol. 46, no. 2, pp. 329-345, 2004.

[46] H. Si, "Tetgen, a Delaunay-based quality tetrahedral mesh generator," ACM Trans. Math. Softw., vol. 41, pp. 11:1-11:36, Feb. 2015.

[47] K. Ko and T. Sakkalis, "Orthogonal projection of points in CAD/CAM applications: an overview," Journal of Computational Design and Engineering, vol. 1, no. 2, pp. 116 - 127, 2014. 
[48] S. Wandzurat and H. Xiao, "Symmetric quadrature rules on a triangle," Computers and Mathematics with Applications, vol. 45, no. 12, pp. 1829-1840, 2003.

[49] M. A. Scott, T-splines as a design-through-analysis technology. Phd thesis, 2011.

[50] B. Jüttler, A. Mantzaflaris, R. Perl, and M. Rumpf, "On numerical integration in isogeometric subdivision methods for PDEs on surfaces," Computer Methods in Applied Mechanics and Engineering, 2016.

[51] G. Strang and G. J. Fix, An analysis of the finite element method, vol. 212. Prentice-Hall Englewood Cliffs, NJ, 1973.

[52] P. Morse and H. Feshbach, Methods of theoretical physics. No. II in International series in pure and applied physics, McGraw-Hill, 1953.

[53] J. E. Cobb, "Tiling the sphere with rational Bézier patches," in TR UUCS-88-009, University of Utah USA, 1988. 